\title{
Quantitative 3D structured illumination microscopy of nuclear structures
}

Felix Kraus ${ }^{1,3}$, Ezequiel Miron², Justin Demmerle ${ }^{2}$, Tsotne Chitiashvili ${ }^{1}$, Alexei Budco ${ }^{1}$, Quentin Alle ${ }^{2}$, Atsushi Matsuda ${ }^{4,5}$, Heinrich Leonhardt ${ }^{1}$, Lothar Schermelleh ${ }^{2}$, Yolanda Markaki ${ }^{1,6 *}$

${ }^{1}$ Department of Biology II, Center for Integrated Protein Science (CIPSM) and Center for Advanced Light Microscopy (CALM), LMU, Martinsried, 82152, Germany

${ }^{2}$ Micron Advanced Bioimaging Unit, Department of Biochemistry, University of Oxford, Oxford OX1 3QU, United Kingdom

${ }^{3}$ Current address: Department of Biochemistry and Molecular Biology, Monash Biomedicine Discovery Institute, 3800, Melbourne, Australia

${ }^{4}$ Advanced ICT Research Institute Kobe, National Institute of Information and Communications Technology, Kobe 651-2492, Japan

${ }^{5}$ Graduate School of Frontier Biosciences, Osaka University, Osaka 565-0871, Japan

${ }^{6}$ Current address: David Geffen School of Medicine, Department of Biological Chemistry, UCLA, Los Angeles, CA 90095, USA

*Correspondence should be addressed to Y.M. (gmarkaki@mednet.ucla.edu) or L.S. (lothar.schermelleh@bioch.ox.ac.uk)

\section{EDITORIAL SUMMARY}

This protocol describes how to prepare samples for labeling nuclei of cultured mammalian cells for three-dimensional structured illumination microscopy of nuclear structures. Image acquisition, registration and downstream image analysis are also described.

\section{TWEET}

Quantitative 3D structured illumination microscopy of nuclear structures @MicronOxford

\begin{abstract}
3D structured illumination microscopy (3D-SIM) is the super-resolution technique of choice for multicolor volumetric imaging. Here we provide a validated sample preparation protocol for labeling nuclei of cultured mammalian cells, image acquisition and registration practices, and downstream image analysis of nuclear structures and epigenetic marks. Using immunostaining and replication labeling combined with image segmentation, centroid mapping, and nearest-neighbor analyses in open-source environments, 3D maps of nuclear structures are analyzed in individual cells and normalized to fluorescent standards on the nanometer scale. This protocol fills an unmet need for the application of 3D-SIM to the technically challenging nuclear environment, and subsequent quantitative analysis of 3D nuclear structures and epigenetic modifications. In addition, it establishes practicable guidelines and open-source solutions using ImageJ/Fiji and the TANGO plugin for highquality and routinely comparable data generation in immunostaining experiments that apply across model systems. From sample preparation through image analysis, the protocol can be executed within one week.
\end{abstract}

Keywords: structured illumination microscopy, 3D-SIM, chromatin, immunofluorescence, nuclear organization, quantitative image analysis 


\section{INTRODUCTION}

The development of super-resolution light microscopy (SRM) techniques over the last decade has extended the range of cell biology into the nanoscale ${ }^{1-4}$. This allows researchers to assess the topology, structure, and dynamics of specifically labeled macromolecules, bridging the gap between light microscopy and structural biology ${ }^{5}$. Linear structured illumination microscopy (SIM, 3D-SIM) is a powerful and versatile method for generating three-dimensional super-resolution data sets of fluorescently labeled biological structures. It provides up to 8-times the volumetric resolution of conventional techniques, deriving from a two-fold improvement in both lateral ${ }^{6,7}$ and axial resolution ${ }^{8,9}$. Rapid multi-channel acquisition of live and fixed specimens and compatibility with widely used chemical and biological labels make it a popular choice among researchers, evident by its multiple commercial and bespoke implementations and hundreds of instruments in use worldwide. However, while studies using 3D-SIM data increase annually, ease of use, reproducibility, and reliable data interpretation is hampered by technical difficulties and undocumented properties of sample preparation, system performance, and multi-channel acquisitions. Established protocols for the production, handling, and analysis of high-quality 3D-SIM data sets are still few and highly application-specific ${ }^{10}$. This gap is particularly acute in respect to the analysis of epigenetic marks and the distributions of proteins and nucleic acids within the 3D volume of the eukaryotic nucleus ${ }^{11,12}$.

Imaging the nucleus is challenging because of the large 3D volume, high density of target epitopes, and optical distortions due to changes in refractive index between the nucleus itself and the surrounding environment. Traditional widefield and confocal microscopy methods continue to be very useful for many aspects of nuclear imaging, but as many of the epigenetic factors being studied interact on spatial scales under the conventional resolution limit of $\sim 200 \mathrm{~nm}$, super-resolution methodologies are required. Within the super-resolution arsenal, the volumetric multi-channel imaging of chromatin necessary to understand nuclear organization is less suited to current super-resolution methods than are other biological areas such as the cytoskeleton, vesicle transport, or viral packaging ${ }^{13}$. While single-molecule localization approaches have revealed fundamental aspects of chromatin organization and enjoy superior spatial resolution ${ }^{14,15}$, they are limited by difficulties in acquiring 3D multi-channel data sets, fluorophore constraints, and the technical complexity of both equipment and sample preparation. Applications of STED microscopy to nuclear and chromatin imaging are rare, perhaps because of the intrinsically low-contrast environment of chromatin, the biological necessity to image more than two channels, and the technical requirement for specialized fluorophores. The benefits of 3DSIM include rapid acquisitions of larger sample volumes, routine acquisition of three (or more) color channels, flexible choice of fluorophores, and operating with and enhancing relative low sample contrast ${ }^{3}$. These specific capabilities combined with a refined immunofluorescence protocol and quantitative data analysis pipeline, facilitate an improved approach to analyzing nuclear targets (nucleic acids or proteins) and epigenetic mechanisms in eukaryotic nuclei.

Here we describe a systematic approach to super-resolution imaging of nuclear and epigenome organization that uses consistent standards in sample preparation, multi-channel alignment, image segmentation, centroid mapping and signal correlation of the resulting data sets. Standardized and robust super-resolution imaging, in concert with diverse complementary techniques to assay nuclear organization (such as multi-C, ChIP-based, and proteomic techniques ${ }^{16}$ ), is essential for creating assays and models of nuclear dynamics and epigenome function with far-reaching mechanistic and diagnostic applications ${ }^{15-19}$.

\section{Development of the protocol}

Protocols for immunofluorescence on cultured mammalian cells are widely available from antibody suppliers, microscope companies, or kept in the institutional knowledge of individual laboratories. However, with the increased resolution and sensitivity of 3D-SIM, certain weaknesses in existing methods have become evident, leading to the development of the current protocol, which is tailored for achieving optimal sample integrity, high signal-to- 
background ratio and minimal sample induced aberration. Existing protocols have been modified in various ways, including: higher-volume washes to reduce background signal; post-fixation to avoid free-floating fluorescent aggregates that can cause artifacts; and equilibration in mounting media to avoid refractive index mismatches in the sample.

Standard acquisition procedures can also introduce errors in the data, so we include 3DSIM-specific instructions for acquiring entire nuclear volumes. Further, the multi-channel alignment, which is critical for precise distance measurement calculations, is frequently insufficient with generally available $2 \mathrm{D}$ calibration samples. To provide a multi-channel calibration sample that reflected the size and optical properties of the nucleus, we developed a protocol for multi-color labeling of replication foci that is used as a channel calibration sample with greater accuracy than conventional methods.

Additionally, we improve existing pipelines, specifically for semi-automated image segmentation and analysis in determining the topology of nuclear structures and correlating distributions, by building routines solely with open-source solutions. While commercial software packages including Volocity, Imaris and Amira have an excellent reputation and user documentation, we focus on freely available, open-source software packages provided by the scientific community. Some of the most popular current tools are, for example, the Mosaic Suite ${ }^{20}$, and GDSC plugins for ImageJ ${ }^{21,22}$, CellProfiler ${ }^{23,24}$ or TANGO ${ }^{25}$. Of these, some are programmed for batch processing of many image stacks, and others offer links to powerful database applications and easy to operate graphical user interfaces for data handling, experiment planning, segmentation, and measurements. The benefits of dedicated software include tailored use and optimization for their specific task, but often require more activation energy from the user, and have reduced flexibility when used for alternative tasks. While most of the evaluations shown in this protocol can be achieved with many available software packages, we choose TANGO here as a demonstration, and have benefitted from its dedicated design for analyzing nuclear structures ${ }^{26,27}$.

\section{Overview of the procedure}

We provide a validated workflow of (immuno-)fluorescence labeling and sample preparation for super-resolution 3D-SIM of mammalian somatic tissue culture and embryonic stem cells, and the subsequent 3D data analysis of labeled sub-nuclear structures. As examples of nuclear structures or processes that benefit from 3D-SIM imaging we generate multi-color data sets of 5-ethenyl-2'-deoxyuridine (EdU) pulse replication-labeled DNA sites, combined with immunolabeling of the replication machinery and/or posttranslational histone modifications, and allocate their precise topology and correlations based on centroid maps of fluorescence intensity peaks. The protocol guides the experimenter through a complete 3D-SIM imaging experiment including relevant biological and technical controls. The workflow encompasses three sections: (1) sample preparation of fixed mammalian cells for antibody-based immunofluorescence; (2) image acquisition of 3D-SIM data and 3D channel alignment with a mammalian nucleus-specific 3D calibration sample of multi-color labeled replication foci; (3) data post-processing and analysis of distances and correlations between two channels of epigenetic markers (histone modifications, and replication sites and machinery), using either the TANGO software package or ImageJ tools (Fig. 1). The sample preparation is designed to maximize labeling specificity and contrast, as well as to minimize effects from optical aberrations. This aspect of the protocol is beneficial for most other imaging methods as well, but is fundamentally important for SIM imaging due to the relative sensitivity of the algorithmic image reconstruction, which produces more detailed images but may also generate systematic artifacts. For optimal measurement accuracy, we introduce a refined 3D alignment method based on biological samples (Fig. 2; Box 1;

Supplementary Fig. 1), and describe detailed workflows for image segmentation and centroid distance calculation for individual- and batch image processing in an open source software environment (Fig. 3; Supplementary Fig. 2). We perform control experiments with fluorescent beads, DNA origami ${ }^{28}$, multi-labeled antibody complexes and directly labeled replicating DNA sites; this provides validated fluorescent standards for registering centroids 
and performing distance measurements and correlation analyses down to $\sim 100 \mathrm{~nm}$ in structural resolution and $\sim 20 \mathrm{~nm}$ localization accuracy (Fig. 4).

\section{Additional applications}

The presented quantitative 3D-SIM imaging pipeline has proven particularly useful to study the mammalian nucleus ${ }^{26,27}$, arguably the most challenging sub-cellular structure to image due to its large size and densely crowded environment. While developed specifically for that context, these workflows can be modified to analyze any sub-cellular organelle of choice by incorporating an organelle-specific label and thus defining an alternate region of interest for the analysis steps. Finally, the sample preparation steps and image analysis procedures can serve as guidelines to a wide range of sample types beyond cultured mammalian cells, including insect cells and few-cell-stage embryos.

\section{Level of expertise required}

The protocol does not require advanced technical knowledge and can be performed by any researcher or student with basic wet-lab technique and microscopy training who is comfortable with handling and operating computer software and image analysis tools. Calibration and maintenance of the 3D-SIM system, however, should be performed in advance by a trained facility expert. Of note, the user must be familiar with the commercial or bespoke SIM system of choice to avoid common errors in image acquisition and reconstruction parameter settings that potentially diminish data quality ${ }^{29}$.

\section{Comparison with other methods}

The emergence of super-resolution microscopy (SRM) has provided great insights into the $3 \mathrm{D}$ organization of the genome at nanometer resolution ${ }^{14,15,19}$. Although 3D-SIM offers less resolution than STED or single-molecule localization techniques such as STORM ${ }^{2}$, it allows the possibility of multi-color super-resolution imaging with conventional fluorophores and mounting media and can accommodate weaker labeling to provide efficient transfer of super-resolution information even at more challenging contrast conditions ${ }^{3,13,30}$. Excellent SIM-specific immunofluorescence and sample preparation protocols are available for the mammalian cytoskeleton ${ }^{31}$, microbes ${ }^{32}$ or live plant cells ${ }^{33}$. However, protocols for immunofluorescent labeling of nuclear factors and their quantification, that specifically address the nuclear environment and multi-channel signal correlations at sub-diffraction resolution, are lacking. We address a current unmet need in focusing on the mammalian nucleus, a target of considerable biomedical importance that is currently underserved by many super-resolution microscopy modalities, particularly compared to applications for visualizing high-contrast structures such as vesicles or microtubules. The complexities of interphase chromatin, histone modifications, replication foci, and other epigenetic marks, which are typically in a spatial scale of $20-200 \mathrm{~nm}$, cannot be resolved with traditional imaging modalities such as widefield or confocal microscopy. Reliance on these techniques may lead to erroneous 'colocalization results' implying functional interactions between targets that may be in reality over $100 \mathrm{~nm}$ apart. However, analysis of intra-nuclear targets with 3D-SIM poses specific challenges due to the large size and dense packing of target structures typically extending over several optical planes. This imposes the inherent risk of much increased (out-of-focus) background fluorescence as well as optical distortions due to refractive index mismatching, and thus a higher potential for reconstruction-based artifacts ${ }^{29}$. For example, the lensing effect, i.e. double/triple structures above the upper edge of the nucleus that become evident if there is a pronounced mismatch between the labeled structure and the surrounding medium, is a frequently encountered phenomenon when imaging the nucleus. Therefore the protocol lays out a framework to achieve high-quality immunofluorescence sample preparation, as well as monitoring image data standards with quality control tools, such as SIMcheck ${ }^{34}$, specifically designed to address 3D-SIMassociated acquisition problems. 


\section{Limitations}

While this protocol is designed to be both flexible and stringent, it has certain constraints. First, the protocol is optimized for investigating the sub-nuclear organization of mammalian cells with high spatial resolution, whereas other specimens and cellular targets may need adjustments from the experimenter to improve temporal resolution or minimize photodamage. Second, the presented protocol focuses on the labeling and imaging of fixed samples. However, application to live-cell experiments is possible using appropriate labeling techniques (e.g., fluorescent protein tags, Halo/SNAP-tag, in vivo replication labeling) and with advanced instrumentation dedicated for fast illumination pattern movement (spatial light modulators or galvo-scanner arrays) and SCMOS camera technology for fast detector readout $^{35-38}$.

The presented analysis workflow relies on high-quality 3D-SIM data with minimal artifacts. Avoidance of imaging artifacts is pivotal for reliable and comparable results and requires optimal sample preparation as outlined in this protocol, as well as optimal system calibration with consistency over time. For instance, the laser beam path, illumination pattern alignment, and optimal matching of the specific sample conditions (e.g., the refractive indices of mounting and immersion media) must meet the system's assumed optical response, encoded by the optical transfer function (OTF) used for the image reconstruction. Mismatching spherical aberration, particularly the case when imaging thick specimens, can cause doubling of structures ('ghosting' or 'lensing') that may erroneously be identified and analyzed as biological structures ${ }^{33,34}$. Furthermore, the level of artefactual high-frequency ('hammerstroke') patterns from reconstructed noise critically depends on the illumination stripe contrast (modulation contrast) in the raw data. The local modulation contrast of the structured illumination pattern can be a limiting factor in data quality, particularly in cases where it is reduced by high levels of out-of-focus blur and 'unstructured' (auto-) fluorescence ${ }^{29,34}$. In this regard, it is pivotal to note the importance of the target of interest; distinct, isolated, and immobile biological structures feature inherently a much higher contrast than, e.g., highly mobile, less affine protein factors with large unbound fractions, or densely packed structures extending along the $z$-axis. These features increase unspecific background and out-of-focus blur contribution, thus reducing modulation contrast and subsequent image quality.

Instruction on all possible systems and the effects on system parameters as well as a systematic description of imaging artifacts is beyond the scope of this protocol and described elsewhere in detail ${ }^{29,33,34}$.

In this protocol, we have optimized image segmentation pipelines that account for some of the complications and meet criteria needed for the analysis of nuclear structures with 3D-SIM data. However, further optimization by the researcher (in both the preparatory and analytical realm) may be required, for example when targets of interest are not welldefined focal structures. Thus, since no protocol can cover the scope of all possible applications, we advise to adapt and modify parts of the guidelines presented here to the needs of each experiment.

\section{Experimental Design}

Considerations for sample preparation. Meticulous sample preparation (steps 1-25) is the key to successful, high-quality 3D-SIM imaging geared towards optimal contrast, labeling specificity, structural preservation, and minimization of optical aberration effects. This requires consideration to the minute details: buffers need to be free from salt precipitates: PBS-based buffers should be preferably filtered and contain $0.05 \% \mathrm{w} / \mathrm{vol}$ sodium azide preventing bacterial contamination; fixation should be efficient yet gentle to maintain ultrastructure. Common hydrophilic fixatives, such as formaldehyde and methanol tend to evaporate quickly. Hence, if the exchange with PBS buffers is not carried out rapidly cells may dehydrate and the sample's structure will be compromised, therefore the sample should never dry out. For 3D-SIM imaging of the nucleus we have found that $2-4 \%$ formaldehyde fixation allows chromatin ultrastructure preservation comparable to live-cell conditions ${ }^{27}$. Harsh coagulating fixatives, such as alcohol, acetone or acetic acid should generally be 
avoided. For applications that require methanol treatment, a preceding formaldehyde fixation will preserve structural and morphological integrity at the 3D-SIM-resolution level (Supplementary Fig. 3). Alternative permeabilization buffers (such as cytoskeletal buffer CSK) can also be well accommodated in 3D-SIM quantitative imaging of nuclear structures ${ }^{39,40}$. Thorough washing after antibody incubations is essential to reduce nonspecific background. Primary antibodies for immunolabeling should have a high affinity to their epitopes and show little, if no, cross-reactivity. Here, suitable controls of antibody specificity, such as Western blot detection, the usage of epitope-free (knock-out) cells, and mock immunostaining with only secondary antibodies, are recommended, while preabsorbed antibodies are preferred in double-immunolabeling experiments. To avoid precipitation we recommend that antibodies are pre-cleared of precipitates by centrifugation and supernatants transferred into fresh tubes. Details of antibodies and conditions used in this protocol can be found in Supplementary Table 1. As extensive photobleaching during imaging can cause reconstruction artifacts, the fluorescent signals should be bright and as photostable as possible. A list of fluorophores well suited for 3D-SIM can be found in Demmerle et al. ${ }^{29}$. However, with regard to contrast and specificity, an optimal signal-tobackground ratio is more important than absolute brightness. After incubation with secondary antibodies, a post-fixation step of $4 \%$ formaldehyde stabilizes antibody complexes and can greatly aid with the elimination of floating fluorescent particles in the imaging process, which may otherwise affect reconstruction quality. The choice of DNA counterstain can also be important, both for establishing an effective nuclear mask during data analysis, or for a more detailed investigation of the chromatin landscape. While most DNA stains tolerate the repeated exposures and photon budget of 3D-SIM imaging, some commonly used dyes are less effective. A representative list of possible DNA counterstains for 3D-SIM imaging is provided in Table 1.

In summary, the optimized sample preparation, DNA counterstain, and immunofluorescence guidelines presented here aim to produce best possible signal contrast with high dynamic range and low background for 3D-SIM investigations of nuclear structure.

TABLE 1 | List of recommended DNA counterstains for 3D-SIM imaging.

\begin{tabular}{llll}
\hline Laser line & Recommended & Alternatives & Not recommended \\
\hline $405 \mathrm{~nm}$ & DAPI & Hoechst 33342 & \\
$488 \mathrm{~nm}$ & SYTOX Green ${ }^{\mathrm{a}}$ & & \\
$561 \mathrm{~nm}$ & & & Propidium iodide \\
$640 \mathrm{~nm}$ & SiR-Hoechst & DRAQ5, SYTOX Red & 7-AAD
\end{tabular}

Note that this list is not exhaustive and recommendations are based on mounting in Vectashield.

${ }^{a}$ Requires additional RNase I treatment

Alignment of 3D-SIM data sets. Multichannel 3D-SIM data, particularly if acquired from multiple cameras, requires post-acquisition color channel alignment. Alignment slides for this calibration can be made by fixing multispectral beads on the plane of the coverslip surface or a 2D array of milled perforations on a mirror-mounted slide. For 2D data sets these slides can correct $x-y$ translations, rotations and zoom variability between detectors, but they fail to address the depth-dependent $z$-shift caused by chromatic dispersion present in multichannel $3 \mathrm{D}$ data sets. Some software packages avoid this by splitting alignments in two phases; first the automated 2D registration using 2D alignment slides, followed by a manual correction in $z$ relying on visual inspection to generate $z$-axis calibration parameters ${ }^{41}$. For preparation of dual-layer bead slides for 3D-channel alignment see the protocol by Demmerle and coworkers published in this issue ${ }^{29}$. In this protocol we outline the preparation of 3D 
alignment slides from EdU incorporation in replicating mammalian cells, followed by a 'click' reaction to couple EdU residues to multiple azide-functionalized fluorophores (Box 1). These slides have the same 3D nuclear foci pattern in all channels, instead of foci on a single z-plane (Fig. 2). When coupled to open-source software capable of iterative 3D registration (e.g. Chromagnon: https//github.com/macronucleus/chromagnon, or similar software ${ }^{42}$ ), these 3D patterns allow the generation of alignment parameters in all spatial dimensions simultaneously. In this way, manual alignment is dispensable, increasing the efficiency and reproducibility of this step. The parameters can then be applied to any other data sets acquired under comparable conditions in a batch-processing mode (Supplementary Fig. 1).

Quantitative image analysis. As the nucleus is a diverse environment with a high degree of spatial organization, and labeling nuclear factors usually results in a high number of signals unsuited for manual analysis, a reproducible and quantitative approach is required. The first step in every image analysis workflow is the rigorous assessment of image data quality, which can be often reduced in the nucleus. With the SIMcheck ${ }^{34}$ plugin suite for ImageJ we have recently introduced a convenient toolset to identify common artifacts and evaluate raw and reconstructed data quality (e.g. photobleaching, signal-to-noise) through objective control parameters (see reference 34 for a detailed description and interpretation guidelines). Reconstructed data sets passing the designated quality thresholds must then be segmented and analyzed in an unbiased and stringent manner, while not imposing unreasonable demands on the researcher for data handling and reproducibility. In the decision-making process for choosing an analytical method, several key considerations should be kept in mind. Firstly, defining the region of interest, or creating a mask, is a critical step in reducing the volume of data to be processed and restricting the analysis to the biologically relevant compartment, such as the nucleus (Fig. 3a). To create nuclear masks, a DAPI acquisition channel is a useful reference signal, but other chromatin references such as H2B-GFP can also be employed. Secondly, defining meaningful parameters for image measurement is essential for subsequent analysis. From the plethora of possible key statistics available for calculation, only a subset is typically used. The most important metrics, besides numbers and volumes of segmented objects, are sub-pixel weighted 3D centroid localizations and the minimal spatial separation between any two centroids of different colors (nearest neighbor distribution). Importantly, with super-resolution microscopy data the classical concept of 'co-localization' loses its utility ${ }^{43}$, as this measure of pixel overlap inherently decreases with increase in optical resolution. We therefore replace typical co-localization analyses with the introduction of minimal distance and (relative) correlation coefficients measurements (Fig. 3d; Fig. 4; Supplementary Table 2). As a first step, these spatial relationships are applied to in vitro and biological standards, such as fluorescent beads or DNA nanorods, to validate distance measurements and help establishing a 'threshold' for anticipated results. Of note is the observed spatial separation of $\sim 90 \mathrm{~nm}$ between fluorescent signals that correspond to dual labeled IgGs against the same epitope (Fig. 4e). In double immunolabeling experiments, the user should therefore anticipate that the spatial separation of signal centroids between the two channels does not normally result in much smaller distances than $\sim 100 \mathrm{~nm}$ (discussed in detail in 27).

Image pre-filtering and watershed segmenting ${ }^{25,44}$ allow an in-depth analysis of fluorescence microscopy data. Here, we use the open source platform ImageJ/Fiji ${ }^{21,45}$ and further apply the TANGO ${ }^{25}$ plugin that is specifically tailored for 3D nuclear images. As compared to standard ImageJ/Fiji analyses, TANGO offers project management architecture and batch processing capabilities for fast and robust image analysis (Supplementary Fig. 2). The implemented pre-segmentation filters identify local intensity maxima and minima to optimally discriminate between adjacent intensity peaks and thus sharpen and enhance features and edges. Post-filtering enables the user to eliminate artifacts from potential oversegmentation. Moreover, measurements that do not meet the anticipated sizes can be predefined as a threshold, allowing the elimination of outliers, such as background signals. Objectivity in the segmentation process is achieved by regularly testing and validating calibration standards of known sizes, such as fluorescent beads or DNA origami (Fig. 4). For 
preparation of DNA origami (nanorods) see Supplementary Method 1. The processing chains described in this protocol have been designed for super resolution 3D-SIM nuclear structure analyses, enabling the application of the same parameters to experimental replicates, facilitating unbiased and rapid data analysis (Supplementary Software).

\section{MATERIALS}

\section{REAGENTS}

- TetraSpeck Microspheres, 0.2- $\mu$ m diameter (Thermo Fisher, cat. no. T-7280)

- Dulbecco's phosphate buffered saline (DPBS, Sigma-Aldrich, tissue culture grade; D8537-500ML)

- Embryomax 0.1\% (w/vol) Gelatin Solution (EMD Millipore, cat. no. ES-006-B)

- Geltrex (basement membrane matrix, Thermo Fisher, cat. no. A1569601)

- $\mathrm{NaCl}$ (Sigma-Aldrich, cat. no. S3014)

- $\mathrm{KCl}$ (Sigma-Aldrich, cat. no. P9541)

- $\mathrm{Na}_{2} \mathrm{HPO}_{4}$ (Sigma-Aldrich, cat. no. S3264)

- $\mathrm{KH}_{2} \mathrm{PO}_{4}$ (Sigma-Aldrich, cat. no. P9791)

- Demineralized $\mathrm{H}_{2} \mathrm{O}$

- Tween 20 (Calbiochem, cat. no. 655204).

- Triton X-100 (Sigma-Aldrich, cat. no. T9284).

- Vectashield, non-hardening antifade mounting medium (Vector Laboratories, cat. no. H1000)

- BSA (Sigma-Aldrich, cat. no. A7906)

- $45 \%$ (wt/vol) fish skin gelatin (FSG, Sigma-Aldrich, cat. no. G7765)

- MAXblock blocking medium (Active Motif, cat. no. 15252)

- 5-Ethenyl-2'-deoxyuridine (EdU, Thermo Fisher cat. no. A10044)

- Sodium azide (Merck, cat. no. 822335). ! CAUTION Sodium azide is a hazardous irritant of skin and eyes. ! CAUTION Sodium azide is very toxic. Wear appropriate protective equipment and work under a fume hood when handling the powder.

- DAPI (Thermo Fisher cat. no. D-1306)

- Chloroform (Sigma-Aldrich, cat. no. 132950). ! CAUTION Chloroform is harmful when inhaled or swallowed; causes skin, eye and respiratory tract irritation. Wear appropriate protective equipment and work under a fume hood.

- Ethanol absolute (100\%; Merck Millipore, cat. no. 100983). ! CAUTION Ethanol is flammable.

- Formaldehyde solution (min. $37 \% \mathrm{w} / \mathrm{vol}$ stabilized with $\sim 10 \%$ methanol; Merck Millipore cat. no. 104003 , or $16 \% \mathrm{w} / \mathrm{vol}$ methanol-free in ampules, Thermo Fisher, cat. no. 28906). ! CAUTION Formaldehyde is carcinogenic and harmful to skin and respiratory tract. Wear appropriate protective equipment and work under a fume hood.

- Methanol (Sigma-Aldrich, cat. no. 494437). ! CAUTION Methanol is flammable and harmful to the skin and respiratory tract. Wear appropriate protective equipment and work under a fume hood.

- Copper (II) sulfate (Baseclick, cat. no. BCMI-004-10)

- Sodium L-ascorbate (Sigma-Aldrich, cat. no. A7631)

- Tris-HCl Buffer (pH 7, 1 M, Sigma-Aldrich, cat. no. T2413)

- Dye-azide (e.g., Alexa Fluor 488 azide Thermo Fisher, cat. no. A10266; or Baseclick 5FAM azide cat. no. BCFA-004-1; or CF568 azide cat. no. 92082)

- Glycine (Sigma-Aldrich, cat. no. G7126)

- Sample of interest. We use EdU pulses to label replication sites combined with immunolabeling of the replication machinery and/or posttranslational histone modifications in C127 cells (ATCC, cat. no. CRL-1804)

- Antibodies for proteins of interest; see Supplementary Table 1 for a list of those we have used 


\section{EQUIPMENT}

- 3D-SIM system (e.g., GE Healthcare OMX; Nikon N-SIM; Zeiss Elyra S.1)

- 6-well plates (BD Falcon, cat. no. 353046)

- Borosilicate precision cover glasses, thickness No. $1.5 \mathrm{H}(170 \pm 5 \mu \mathrm{m}) ; 18 \times 18 \mathrm{~mm}$ or 22x22 mm (Marienfeld Superior, cat. no. 0107032)

- Fine-tip forceps (Dumont \#5; Fine Science Tools, cat. no. 11251-20 or similar)

- Humidified chamber

- Transparent nail polish (top coat), or CoverGrip coverslip sealant (Biotium, cat. no. 23005)

- Syringe filter, 0.2-0.45- $\mu \mathrm{m}$ pore size (Sartorius, cat. no.17598)

- Clean glass microscope slides $(76 \times 26 \mathrm{~mm} /$ cleaned packed in fiber-free boxes)

- $50 \mathrm{ml}$ tubes (BD Falcon, cat. no. 352070)

- Low-protein-binding 1.5-ml tubes with Safe-Lock lid (Eppendorf, cat. no. 022431081)

- Vacuum aspirator

- 3-ml transfer pipettes (Pastettes, Alpha Laboratories, cat. no. LW4040, (Falcon, cat. no. 357524). A CRITICAL We recommend the use of disposable plastic Pastettes for applying solutions and washing buffers in the plastic dishes for ease of use and speed of transfer.

- Soft tissue paper (Kimwipe, Kimberly-Clark Professional, cat. no. 34155)

- Cotton-tipped swabs for optical cleanup (Edmund Optics, cat. no. 56-926)

- Lens-cleaning tissues (Whatman, GE Healthcare, cat. no. WHA2105862)

- Slide storage boxes (Pelco, cat. no. 2106)

- Parafilm (VWR, cat. no. 52858-000)

- Computer; Unix or Windows 64-bit OS is preferred, with 32-bit systems acceptable but likely to show slow performance. Adequate storage capacities for the database setup are required, as well as 4 to 8 GB RAM and current multi-core processors

- The Fiji distribution ${ }^{45}$ of Image $J^{21,22}$ is recommended. Other ImageJ distributions must have the BioFormats Importer $^{46}$ installed. All distributions should be the most up-to-date version.

- The SIMcheck plugin ${ }^{34}$ for ImageJ/Fiji should be installed for quality control and assessment of raw and reconstructed 3D-SIM data.

- The TANGO plugin ${ }^{25}$ if no image analysis software is already in use or capable of replicating all morphological and statistical operations.

REAGENT SETUP

20x PBS 20x PBS is $160 \mathrm{~g} \mathrm{NaCl}, 4 \mathrm{~g} \mathrm{KCl}, 28.8 \mathrm{~g} \mathrm{Na}_{2} \mathrm{HPO}_{4}, 4.8 \mathrm{~g} \mathrm{KH}_{2} \mathrm{PO}_{4}$, pH 7.4; for working dilutions, dilute with demineralized $\mathrm{H}_{2} \mathrm{O}$ to make $1 \times \mathrm{PBS}$.

1x PBS Add $10 \mathrm{ml}$ of $20 x$ PBS buffer to $190 \mathrm{ml}$ demineralized $\mathrm{H}_{2} \mathrm{O}$ to obtain $200 \mathrm{ml}$ of $1 x$ PBS buffer. Store the buffer at room temperature $\left(23^{\circ} \mathrm{C}\right)$ for up to $6-12$ months.

Sodium azide solution (optional) Add $5 \mathrm{~g}$ of sodium azide to $100 \mathrm{ml}$ demineralized $\mathrm{H}_{2} \mathrm{O}$ to obtain a $5 \% \mathrm{w} / \mathrm{vol}$ sodium azide solution. This solution can be used as a supplement to PBS to prevent bacterial contaminations. When making sodium azide supplemented PBS add $10 \mathrm{ml} 20 x$ PBS buffer and $10 \mathrm{ml}$ sodium azide solution to $180 \mathrm{ml}$ demineralized $\mathrm{H}_{2} \mathrm{O}$ to obtain $200 \mathrm{ml} 1 \mathrm{x}$ PBS buffer. Store at room temperature for up to 6-12 months.

Fixation solution Freshly prepare $2-4 \%$ (vol/vol) formaldehyde from stock in PBS. According to the number of samples to be fixed dilute the corresponding amount of formaldehyde stock solution with tissue culture grade PBS to obtain the desired fixative concentration. E.g., for fixation on a coverslip in a 6 -well multiwell plate with $2 \%$ formaldehyde solution add $0.25 \mathrm{ml}$ of $16 \%$ (wt/vol) formaldehyde stock solution to $1.75 \mathrm{ml}$ PBS. Mix well and use immediately.

Postfixation solution Freshly prepare 4\% (vol/vol) formaldehyde in PBS and use immediately. 
Washing buffer (PBST, 0.02\% Tween 20 in PBS) Dissolve $200 \mu \mathrm{l}$ Tween 20 to 1 liter of PBS. The buffer can be stored at room temperature for several months.

$10 \% \mathrm{vol} / \mathrm{vol}$ Triton X-100 stock solution Add $10 \mathrm{ml}$ of Triton X-100 to $90 \mathrm{ml}$ of PBS.

Dissolve overnight by stirring. Buffer can be stored at room temperature for several weeks or at $4{ }^{\circ} \mathrm{C}$ for several months.

Permeabilization buffer Add $5 \mathrm{ml}$ of $10 \%$ Triton X-100 stock solution, $95 \mathrm{ml}$ PBS. Mix by stirring. A range between $0.1-0.5 \%$ of Triton $X-100$ can be used depending on the antibody accessibility requirements and cell type. Buffer can be stored at room temperature for several weeks or at $4{ }^{\circ} \mathrm{C}$ for several months.

Blocking solution Add $2 \mathrm{~g}$ of BSA and $1.2 \mathrm{ml}$ of $45 \% \mathrm{w} / \mathrm{vol}$ fish skin gelatin to $100 \mathrm{ml}$ PBST and mix by stirring. Filter through a $0.45 \mu \mathrm{m}$ syringe filter. Buffer can be stored at $4{ }^{\circ} \mathrm{C}$ for several days or at $-20^{\circ} \mathrm{C}$ for several months. Alternatively use MAXblock.

Quenching solution PBS, $20 \mathrm{mM}$ glycine prepared freshly. Add glycine powder to PBS, dissolve thoroughly and use immediately. $\triangle$ CRITICAL All buffers can be made with demineralized water but when possible ultrapure water is recommended.

DAPI solution Make a DAPI stock solution of $0.5 \mathrm{mg} / \mathrm{ml}$ in PBS. Store stock solution at $20{ }^{\circ} \mathrm{C}$ for up to a year and protect it from light. DAPI has poor solubility in aqueous solutions so sonication might be necessary. Dilute the stock solution to a working concentration of $2 \mu \mathrm{g} / \mathrm{ml}$ in PBST. For preparing $10 \mathrm{ml}$ of DAPI solution, add $40 \mu \mathrm{l}$ of DAPI stock solution to $10 \mathrm{ml}$ PBST. Vortex the mixture well and use it within a few hours. Protect it from light. $10 \mathrm{mM}$ EdU stock solution Add $50 \mathrm{mg}$ of EdU to $19.84 \mathrm{ml}$ of DMSO. Dissolve the solution by vortexing and store it at $-20^{\circ} \mathrm{C}$ for several months.

0.5 M Sodium L-ascorbate solution For $1 \mathrm{ml}$ of solution, add $99.05 \mathrm{mg}$ of sodium L-ascorbate to $1 \mathrm{ml}$ demineralized $\mathrm{H}_{2} \mathrm{O}$. Dissolve the solution by vortexing and store at $-20^{\circ} \mathrm{C}$ for several months.

$50 \mathrm{mM} \mathrm{CuSO}{ }_{4}$ solution Add $10 \mathrm{mg}$ of $\mathrm{CuSO}_{4}$ to $1.25 \mathrm{ml}$ of demineralized $\mathrm{H}_{2} \mathrm{O}$. Dissolve the $\mathrm{CuSO}_{4}$ by vortexing and store the solution at $4{ }^{\circ} \mathrm{C}$ for several months.

EdU click solution containing $100 \mathrm{mM}$ Tris/ $/ \mathrm{HCl} \mathrm{pH} \mathrm{7,} 5 \mathrm{mM} \mathrm{CuSO}{ }_{4}, 50 \mathrm{mM}$ sodium Lascorbate, $20 \mu \mathrm{M}$ dye-azide in $\mathbf{d d H}_{2} \mathrm{O}$ For $1 \mathrm{ml}$ of EdU click solution, add $100 \mu \mathrm{l} 1 \mathrm{M}$ Tris- $\mathrm{HCl}, \mathrm{pH} 7,100 \mu \mathrm{l}$ of $50 \mathrm{mM} \mathrm{CuSO}_{4}, 100 \mu \mathrm{l} 0.5 \mathrm{M}$ sodium L-ascorbate, $200 \mu \mathrm{l} 0.1 \mathrm{mM}$ dye-azide and $500 \mu \mathrm{l}$ of demineralized $\mathrm{H}_{2} \mathrm{O}$. Prepare the solution fresh and protect it from light.

\section{EQUIPMENT SETUP}

Preparing coverslips for cell seeding Thoroughly clean coverslips with a Kimwipe soaked with $80 \%$ ethanol to remove dust and sterilize by immersing in $100 \%$ ethanol where they can be stored. We recommend not to flame or autoclave the coverslips for sterilization, as they deform. Under a laminar flow, transfer coverslips with forceps from the ethanol container and initially place vertically into 6-well multiwell dishes (against the well's wall), until all ethanol has evaporated. $\triangle$ CRITICAL Make sure coverslips are well dried before flipping them horizontally and adding the cell suspension.

Humidified dark chamber To prepare the chamber place a strip of Parafilm well attached onto a smooth surface (e.g. plastic or glass) within the chamber (e.g. made from a circular $15 \mathrm{~mm}$ plastic dish or opaque plastic or metal box) and line with PBS- or water-soaked filter paper.

Structured illumination microscopy requirements The 3D-SIM system should be in good working order and regularly maintained by an imaging professional or technically familiar researcher, in cooperation with the instrument provider's instructions. Further instrument alignment procedures, and performance checks with 3D-SIM-specific calibration materials and procedures should be performed as necessary ${ }^{29}$. For multi-camera systems, a channel alignment sample is required ${ }^{47}$. A protocol for the preparation of biological calibration slides for 3D alignment of 3D-SIM data sets is presented in Box 1. Constant room temperature is a fundamental pre-requisite for consistent acquisitions and minimization of mechanical drift as well as camera output. Constant room temperature $\left(23^{\circ} \mathrm{C} \pm 5{ }^{\circ} \mathrm{C}\right.$, $<0.5^{\circ} \mathrm{C}$ variation/h) is recommended for consistent acquisitions and minimization of 
mechanical drift or changes in camera output. Laser power and camera exposure times should be adjusted to achieve the optimal trade-off between high dynamic range and acceptable photobleaching. Exposure times for fixed cell applications should typically be longer than $15 \mathrm{~ms}$ to reduce intensity fluctuations but not exceed $100 \mathrm{~ms}$ to avoid potential drift artifacts.

\section{PROCEDURE}

\section{Preparation of adherent cells or embryonic stem cells (ESCs) for immunofluorescence TIMING $7 \mathrm{~h}+12-24 \mathrm{~h}$ cell seeding and growth}

1) Seed cells approximately $24 \mathrm{~h}$ prior to fixation on pre-cleaned coverslips under appropriate conditions (typically $5 \% \mathrm{CO}_{2}, 37^{\circ} \mathrm{C}, 95 \%$ relative humidity). For ESCs seeding, use gelatin or Geltrex coated coverslips. Coat coverslips by adding $1-2 \mathrm{ml}$ of gelatin solution per well preferably overnight. For Geltrex coating add $1 \mathrm{ml}$ solution and incubate for $30 \mathrm{~min}$ at $37^{\circ} \mathrm{C}$ followed by quenching for $30 \mathrm{~min}$ at RT.

$\triangle$ CRITICAL STEP Seeding densities at this step will vary according to the application. For imaging of individual cells, required when imaging the nucleus, confluency should not exceed $70 \%$ to reduce stray light from neighboring cells. If a label that must be introduced under live-cell conditions (e.g. EdU, MitoTracker) is required, it should be added here at the appropriate concentration and duration. For stem cell staining ensure seeding of single cells in order to produce small colonies that will facilitate imaging and reconstruction quality.

? TROUBLESHOOTING

2) Remove medium with a pipette tip attached to the hosing of a vacuum pump and wash cells two times with PBS (tissue culture grade).

$\triangle$ CRITICAL STEP Do not use PBS containing sodium azide prior to fixation, as it is toxic to the cells.

3| Place coverslips cell-side up into fresh 6-well plates containing fixation solution and incubate for $10 \mathrm{~min}$ at room temperature.

! CAUTION Formaldehyde and Methanol are carcinogens. Wear appropriate protective equipment and work under a fume hood.

$\triangle$ CRITICAL STEP Before fixation, verify the fixation reagent is compatible for labeling of the target structure. Certain antibody-epitope detections, for example PCNA immunostaining, require post-fixation and permeabilization with methanol (Supplementary Fig. 3).

? TROUBLESHOOTING

4| Gently remove the fixative with the vacuum while simultaneously adding washing buffer with a Pastette for 4-8 s, ensuring the coverslips are always submerged in solution.

$\triangle$ CRITICAL STEP Stepwise fixative exchange with washing buffer ensures that cells do not dry out and cellular structures are optimally preserved after formaldehyde cross-linking. This is achieved by the progressive exchange of fixative with washing buffer that contains Tween-20, a surfactant that decreases surface tension. When working with ESCs make sure the vacuum is very gentle to avoid detachment.

5| Wash twice with washing buffer.

$\triangle$ CRITICAL STEP We recommend washing the coverslips in multi-well plates and exchanging the washing buffer with the help of a vacuum pump. Always add the buffers to the side of the well and not directly onto the cells to avoid cell detachment from the coverslip. If working with delicate cells, mitotic cells or stem cells that grow in colonies and are easily detached, we recommend the use of a very gentle vacuum. Alternatively, we recommend blotting the coverslip edge on a tissue to remove excess liquid, and slowly 
dipping into a $50 \mathrm{ml}$ beaker of washing solution. This procedure can be repeated several times with a series of beakers.

6 Quench free aldehydes by incubating for 10-20 min in quenching solution at room temperature (optional).

7| Wash twice with washing buffer.

PAUSE POINT Samples may be left in multi-well plates containing $1 \times \mathrm{PBS}$ at $4^{\circ} \mathrm{C}$ overnight.

8 Exchange washing buffer with $2 \mathrm{ml}$ of permeabilization solution, and incubate for $10 \mathrm{~min}$ at room temperature. When methanol treatment is required, fix and permeabilize by adding ice-cold methanol for $5 \mathrm{~min}$. Stepwise exchange methanol with washing buffer as described in step 4.

$\triangle$ CRITICAL STEP Do not omit initial formaldehyde fixation step when methanol fixation is implemented. Methanol extraction should only be done after an initial cross-linking with fixation solution in order to optimally preserve nuclear features as shown in Supplementary

Figure 3. Incubation in permeabilization buffer is not usually necessary when using methanol fixation/extraction, but if the user finds that the antibody accessibility is improved then it can be performed.

9 Place a clean Parafilm in a humidified chamber and apply $100 \mu \mathrm{l}$ of blocking solution in drops. Remove coverslips from permeabilization solution, blot dry by touching edge on a clean tissue, and place gently, cell side down, on the drop of blocking solution.

10| Incubate in blocking solution for 30-60 min at room temperature.

11| Dilute primary antibodies of choice in blocking solution to the appropriate concentration to make primary antibody solution.

$\triangle$ CRITICAL STEP Transfer primary antibodies on ice.

? TROUBLESHOOTING

12 Place clean Parafilm in a humidified chamber and apply at least $50 \mu \mathrm{l}$ of primary antibody solution in drops. Remove coverslips from blocking solution, blot dry by touching edge on a clean tissue, and place gently, cell side down, on a drop of primary antibody solution. Incubate for $1 \mathrm{~h}$. If cells have been pulsed with EdU, incubate with EdU cocktail solution first for $30 \mathrm{~min}$, wash as in Step 2 and then proceed with primary antibody incubation at room temperature.

13 Remove coverslips from primary antibody solution, blot dry by touching edge on a clean tissue and place coverslips cells side up into 6-well plates containing washing buffer. Wash coverslips four times with washing buffer.

14| Dilute secondary antibodies of choice in blocking solution to the appropriate concentration to make secondary antibody solution.

$\triangle$ CRITICAL STEP Transfer secondary antibodies on ice and protect from light. ? TROUBLESHOOTING

15 Place clean Parafilm in a humidified container and apply at least $50 \mu \mathrm{l}$ of secondary antibody solution in drops. Proceed with secondary antibody incubation as in Step 12.

16| Remove coverslip from secondary antibody solution and wash four times with washing buffer. 
17| Remove coverslip from the well of washing solution and place coverslips, cell side up, into wells containing at least $2 \mathrm{ml}$ of post-fixation solution.

18| Incubate in post-fixation solution for $10 \mathrm{~min}$ at room temperature.

! CAUTION Formaldehyde and Methanol are carcinogens. Wear appropriate protective equipment and work under a fume hood.

19| Wash as in Step 4.

20| Transfer coverslips to wells containing 1-2 $\mathrm{ml}$ of chromatin counterstain solution, if desired. For counterstaining with DAPI solution, incubate for $5 \mathrm{~min}$ at room temperature.

21| Wash three times with PBS.

22 On a piece of clean Parafilm, apply at least $30 \mu \mathrm{l}$ of mounting solution in drops and lower coverslip cell-side down onto the drop. Incubate for $5 \mathrm{~min}$ at room temperature.

$\triangle$ CRITICAL STEP The mounting solution may vary depending on the application, but mounting solutions containing counterstain should be avoided.

23 Apply 10-15 $\mu \mathrm{l}$ of mounting reagent on the unfrosted side of an appropriately labeled slide. Mounting on the frosted side will affect the levelness at different ends of the slide causing a tilt relative to the focal plane. Positioning of the coverslip may vary depending on the configuration of the microscope stage, but often it is required that the coverslip is placed in the center of the slide.

24| Remove the coverslip from the drop of mounting reagent, blot dry by touching edge on a clean Kimwipe to remove excess mounting reagent, and gently lower the coverslip down onto the drop, avoiding any bubbles. Gently remove excess mounting medium by blotting over the coverslips with a Kimwipe or soft tissue.

$\triangle$ CRITICAL STEP Avoiding refractive index aberrations in the sample is critical. Preincubation in mounting reagents avoids dilution of the final mounting condition, and extra care to avoid introducing air bubbles into the mounting solution upon application of the coverslip must be taken.

25| Allow coverslips to settle for a few minutes and then seal thoroughly with an appropriate amount of sealant. Slides may be taken immediately to the microscope or stored until imaging.

A CRITICAL STEP Allow sealant to air-dry before storage. The choice of sealant may vary, and should be tested empirically. Commercially available nail polish is generally sufficient, and formulations with color or quick-hardening qualities often provide a longer-lasting seal. Repeated exposure to EtOH may degrade the seal, which can be reapplied at any point in the lifetime of the sample.

PAUSE POINT Slides may be stored in slide storage boxes for a few weeks at $4^{\circ} \mathrm{C}$ in the dark. For long-term storage samples can be placed at $-20^{\circ} \mathrm{C}$.

$\triangle$ CRITICAL STEP Do not place samples at $-20^{\circ} \mathrm{C}$ between imaging rounds as great temperature variations result in floating particles and compromised sample and image and reconstruction quality.

? TROUBLESHOOTING 


\section{Box 1 | Preparation of biological calibration slide for 3D alignment of 3D-SIM data sets TIMING 6 h + 12-24h cell seeding and growth}

For experiments that involve multiple channels prior to imaging the specimens, acquire images of the biological calibration slide first and proceed with alignment as described below.

\section{Procedure}

1. Follow Step 1 from the main PROCEDURE.

2. For pulse-labeling, add $10 \mu \mathrm{l}$ of EdU stock solution to $10 \mathrm{ml}$ of fresh media, replace prior media with EdU-containing media, and incubate for desired pulse-time ( 10-15 min).

\section{Follow Steps 2-8 from the main PROCEDURE.}

4. Make suitable volume of EdU click solution (EdU cocktail) for the number of coverslips required ( $\sim 50 \mu \mathrm{l}$ per coverslip, see REAGENTS SETUP). A ratio of 7:3:2:1 for CF405M-, Alexa Fluor 647-, CF568 or Alexa Fluor 594- and Alexa Fluor 488-azide, respectively, is suitable to provide high dynamic range and sufficiently low photobleaching for a multiple channel EdU-labeled sample. Dependent on the system configuration and photochemistry of the respective dyes other ratios may have to be empirically determined. Note that for a balanced labeling it is critical to incubate all dyes simultaneously.

5. Incubate cells with the EdU cocktail for $30 \mathrm{~min}$ in the dark at room temperature and wash as in Step 2 of the main PROCEDURE.

6. Follow steps $17-25$ of the main PROCEDURE for the preparation of adherent cells, but omitting Step 20 , as chromatin counterstain would mask foci signal in that wavelength.

7. Follow Steps 26-31 of the main PROCEDURE for image acquisition and reconstruction. Approximately $1 / 3$ of cells in the sample should have undergone $S$ phase during the EdU pulse. We recommend acquisitions from cells whose foci pattern is of mid-late $S$ phase (Fig. 2) as these foci are distinct, high-contrasted and distributed evenly throughout the entire nuclear periphery and around nucleoli. Early $S$ phase patterns are still punctate but tend to result in a more diffuse, low-contrast signal throughout the nuclear volume, making them a more challenging sample for image registration.

$\triangle$ CRITICAL STEP Ensure that all channels are acquired with similar signal-to-noise levels.

8. For 3D alignment use the commercial system's image registration, or open-source software solutions such as Chromagnon (https//github.com/macronucleus/chromagnon). The latter provides adaptive correction for translation in $x, y$ and $z$ direction, rotation and magnification by measuring an image correlation parameter (Supplementary Fig. 1). We recommend using the central wavelength as reference for all other channels.

Image acquisition and reconstruction TIMING variable: setting up and validating instrument and reconstruction parameter (0.5-2 h); image acquisition (1-5 min per data set); reconstruction and alignment (2-10 min per data set)

26| Before starting the image acquisition, check to ensure that system parameters (laser line/power, objective, filter sets, camera status, frame size, channel acquisition order, $z$-sectioning distance, camera mode/speed) are appropriate for the application and that the system is properly calibrated and free of any distortions in the optical path. This should be done with the assistance of an expert microscopist or high-level user. 
$\triangle$ CRITICAL STEP For imaging the biological calibration slide make sure that the acquisition parameters match those of the samples in terms of image size, $z$-depth, and camera selection.

27| Before mounting samples slides on the microscope, clean the coverslip surface with $80 \% \mathrm{EtOH}$ followed by chloroform, using an adhesive-free cotton swab or optical tissue. If required wipe dry the objective lens from residual immersion oil with optical tissue followed by wiping with a chloroform-soaked cotton swab.

$\triangle$ CRITICAL STEP Clean coverslip by wiping straight across and avoid contacting sealant.

28| Mount the slide on the microscope with the appropriate immersion oil, taking care not to introduce bubbles into the oil. For the use of oil objectives without correction collar, it is recommended to select immersion oil with a refractive index that provides the best match of the optical condition in the sample and the OTFs used for the reconstruction. Oil selection can be calculated with a number of approaches ${ }^{33,34}$. The selection takes into account the wavelength and imaging depth optimum and the refractive index of sample and mounting medium at a given temperature, aiming to reduce spherical aberration mismatch artifacts. As a general rule we recommend to increase the refractive index of the immersion oil with increased sample thickness or imaging depth, respectively ${ }^{29}$. Alternatively, if using a silicone or oil objective with correction collar, the same effect can be achieved with appropriate adjustments of the collar position.

29| After finding a midsection plane of the cell, adjust exposure times and laser power to utilize as much bit depth of the camera as recommended, while avoiding saturation and minimizing photobleaching.

$\triangle$ CRITICAL STEP All subsequent analysis will depend on the quality of the raw data. Each sample type and combination of labels will require optimization of exposure and illumination settings.

30| As a general guideline, we recommend to acquire one to two empty image planes above and below the structure of interest (i.e., the DAPI-stained nucleus), that do not show any stripes but only unstructured out-of-focus blur. Typically, no more than $0.5 \mu \mathrm{m}$ must be added above and below the nucleus. Avoid imaging excessive numbers of $z$-planes that do not contain desired information to minimize acquisition time, data accumulation and photobleaching and to ensure optimal image reconstructing and data quality in the post-processing steps.

31 Process raw data with the appropriate commercial or open source image reconstruction software ${ }^{42,48}$. Make note of all settings in the software. We strongly recommend the use of channel-specifically measured optical transfer functions (OTFs) where possible rather than synthetic OTFs ${ }^{29}$. Use SIMcheck ${ }^{34}$ to assess reconstructed data quality and adjust settings accordingly.

$\triangle$ CRITICAL STEP Reconstruction artifacts will be segmented and analyzed along with real signal in post-processing steps. Each sample type and combination of labels may require optimization for OTF, background cut-off, and filter settings.

32 Cut-off values below the modal intensity of the reconstructed 3D-SIM image stack and convert to a 16-bit TIFF stack in Fiji-e.g., using the SIMcheck 'Threshold \& 16-bit Conversion' utility ${ }^{34}$.

PAUSE POINT Image stacks can be used to create representative images of the experiment. 


\section{Quantitative image analysis with ImageJ/Fiji TANGO plugin Defining the nuclear volume TIMING 1-2 min / image stack}

33| The first step in image analysis is to introduce processing chains to create a mask of the nucleus and define the nuclear volume. We recommend the use of processing chains developed for 3D-SIM data sets, which can be used by importing the provided templates in Supplementary Software. An overview of these templates is shown in Supplementary Table 3. For alternative single file analysis with standard ImageJ/Fiji refer to Supplementary Method 2. A Readme file, containing a quick installation guide for both TANGO and MongoDB can be found in the Supplementary Software.

$\triangle$ CRITICAL STEP For TANGO analyses, the nuclear stain (for example DAPI) must be the first channel in the composite image stack.

34| Add prefilters by choosing 'Gaussian 3D (IJ)' filter from the 'Misc 3D Filters' dropdown menu. ' $x$ ', ' $y$ ' and 'z' parameters should be set to 2 .

35| For segmentation of the nucleus use either 'Nucleus Edge Detector' or 'Hysteresis Segmenter' and select the 'OTSU iterator' set to 'Auto Threshold'. Include minimal size restrictions (> 5000 voxels) to avoid subsegmentation of the nuclear volume.

\section{? TROUBLESHOOTING}

36| Add post-filters and choose 'Morphological Filters 3D' from dropdown menu. Use the 'Fill Holes 2D' method.

37| Add 'Binary Close' method, set the $x y$ radius to 10 and check 'Use Image Scale for z-radius'.

38| Add 'Fill Holes 2D' method.

\section{? TROUBLESHOOTING}

39| Choose the 'Size and Edge Filter' from the dropdown menu. Set minimal voxel size for structure to 10,000 .

$\triangle$ CRITICAL STEP Defining a reasonable lower cutoff for the nucleus ensures smaller objects, such as stained precipitates, are not used in the follow-up structure segmentation and measurements.

40| Save the processing chain under a name of choice and set it as a segmentation routine for the nucleus or compartment of interest.

41| In the 'Data' tab, check the options 'Process Nuclei' and 'Crop Cells' and run the mask segmentation.

$\triangle$ CRITICAL STEP Selection of an appropriate segmenting algorithm is critical to accurate and robust image analysis. Therefore, we advise the user to invest time in this step, allowing testing and benchmarking of the suitable segmentation routine. The calibration standards

(Fig. 4) are a good basis for this initial benchmarking.

\section{Definition of Measurements TIMING 5 min}

$\triangle$ CRITICAL STEP Defining useful measurements of image data is essential for efficient data analysis. All segmented objects generated by the processing chains in TANGO (Steps 42-51) can be measured regarding various parameters depending on experimental needs. Useful readouts include $x y z$ coordinates of intensity gravity centers (centroids), which are the basis of nearest neighbor minimal distance calculations, or to obtain spatial distribution patterns of two distinct labeled nuclear proteins of interest. Measurement of volumes can be used for recording dynamic nuclear changes over time or under various cellular conditions. 
42 In the 'Edit Experiment' tab, select 'Measurements', add a measurement from the drop-down list, such as minimal distances between nearest neighbors (NN; using 'Minimal Distances'), or volumes and $x y z$ coordinates (using 'Measure Geometric Simple'). Click 'Edit' to adjust the fine parameters; e.g., select the structure to be measured. Repeat the setup for measurements of different structures and calculations. Save selected measurements with clicking on 'Save Changes'.

? TROUBLESHOOTING

\section{Structure segmentation with the ImageJ/Fiji TANGO plugin $\bigcirc$ TIMING 3 min per image} stack, depending on image size and number of z-slices

43| To define the structure of interest for volumetric analysis, select the corresponding region of the image stack with the use of appropriate filters and segmentation algorithms

(Fig. 3, Supplementary Fig. 2). For focal signals (e.g., short EdU pulses) use the 'Focal_Spot' processing chain; for extended structures such as long EdU pulses or immunolabeled histone marks, use the 'Volumetric-Spot' processing chain. The respective processing chain templates are provided in the Supplementary Software and in Supplementary Table 3.

44| Add a pre-filter by choosing from the dropdown menu of the 'Fast Filters 3D' the TopHat filter. Parameters should be set to 1 for xyz. This step will enhance the contrast of the structures of interest.

45| Add a pre-filter by choosing from the dropdown menu of the 'Misc 3D Filters' the LoG (Laplace of Gaussian) filter. Parameters should be set to 2 for xyz. LoG operations emphasize edges between an object and the surrounding background. This allows sharper edge detection during the segmentation process (Fig. $\mathbf{3 b}$ ).

? TROUBLESHOOTING

46| Choose 'Spot Detector 3D' as a method for segmenting focal image signals. This method will use a watershed algorithm for separating foci in 3D from each other and from the background, leading to better focal segmentation and detection of high resolution image signals (Fig. 3b) compared to only intensity based approaches ${ }^{25,44}$. As a foreground/background threshold method, use AutoThreshold and the Otsu iterator.

47| Use the Otsu method for the background limit and seed threshold. Set seeds (segmenting objects) to an upper Hessian limit of $95 \%$ of bright pixels, reducing the risk of segmenting background or nonspecific signals.

\section{? TROUBLESHOOTING}

48 Choose the 'Erase Spots' method from the 'Post-Filters Menu' dropdown menu and check the box to use the filtered image. Set number of criteria to 1 and define \#1 criteria as signal-to-noise Ratio (SNR). Set SNR value between 1.5 and 2.

$\triangle$ CRITICAL STEP Depending on the image quality, the SNR value can be adjusted in the filter options.

49| Set 'Intensity Estimation' to mean intensity and 'Background Estimation' to whole nucleus.

50| Choose the 'Size and Edge Filter' from the dropdown menu. Set minimal voxel size for structure to 2 .

51| Save the Processing Chain under a name of choice and use this chain as the segmentation routine for the nuclear structure under investigation. 
52| In the 'Data' tab, select the cells of interest, check the option 'Process Structures' and run the segmentation.

\section{Measurements of image structures TIMING 2-5 min}

53| Following structure segmentation, check the 'Measurement' box in the cell field and press 'Run'.

$\triangle$ CRITICAL STEP If the 'Override Measurements' box is checked, analysis of the data set performed prior will be overwritten.

\section{Data export $\bigcirc$ TIMING 5 min}

54| Export measurements by clicking the 'Export Measurements' bottom in the 'Data' tab. The program will save .xls files for each channel in a dedicated folder. .xls files are compatible with standard spreadsheet software (Excel, LibreOffice) and programming/scripting languages ( $R$, Python, Matlab and others).

$\triangle$ CRITICAL STEP Export all measured parameters for all measured channels.

\section{Visualization and plotting (data curation) $\bigcirc$ TIMING $\sim 30 \mathrm{~min}$ to $1 \mathrm{~h}$}

55| Generate histograms of measured data using the 'Mathematics Add-In' in Excel (after installation, switch to the 'Data' tab and select 'Data Analysis'), GraphPad Prism or use R (a user-friendly online tool for boxplots ${ }^{49}$ can be found at http://shiny.chemgrid.org/boxplotr/). For plotting correlation coefficients scatter plots are also very useful for a visual comparison of different populations. For a comparison of the resulting analyses to validated technical and biological standards refer to results in Figure 4 and Supplementary Table 2. For applications regarding nuclear structures and epigenetic factors refer to Figure $\mathbf{5}$ and the literature ${ }^{26,27}$.

$\triangle$ CRITICAL STEP When plotting measurements such as minimal distances, attention should be paid to detecting and excluding outliers. Generally these values range beyond the mean plus two-fold the standard deviation (SD). More sophisticated statistical solutions, however, can be employed as well as empirical and rational filtering of the data, which are dependent on the experimental data, structure of choice, and number of specimens used for the study.

\section{? TROUBLESHOOTING}

Troubleshooting advice can be found in Table 2.

\section{TIMING}

The timing for quantitative image analysis (Steps 33-55) is dependent on the number and size of image data. The timing below is based on estimates for $512 \times 512$-pixel (px) 3-color image stacks including $65 z$-slices (8- $\mu$ m nucleus thickness).

Step 1, preparation of adherent cells or embryonic stem cells for immunofluorescence: 12 $24 \mathrm{~h}$ (or according to preferred cell growth conditions)

Steps 2-25, preparation of adherent cells or embryonic stem cells for immunofluorescence: $\sim 7 \mathrm{~h}$

Steps 26-32: image acquisition and reconstruction: $\sim 4 \mathrm{~h}$ per sample

Steps 33-51: definition of the nuclear volume: $\sim 1 \mathrm{~h}$ for 10 image stacks

Steps 52-55, measurement of image structures: $\sim 3 \mathrm{~h}$ for 10 image stacks

Box 1, preparation of biological calibration slide for 3D alignment of 3D-SIM data sets: $6 \mathrm{~h}+$ 12-24 $\mathrm{h}$ cell seeding and growth 
TABLE 2 | Troubleshooting table.

\begin{tabular}{|c|c|c|c|}
\hline Step & Problem & Possible reason & Solution \\
\hline \multirow[t]{2}{*}{1} & \multirow[t]{2}{*}{$\begin{array}{l}\text { Cells detach or grow at } \\
\text { the edges of the } \\
\text { coverslips }\end{array}$} & \multirow[t]{2}{*}{$\begin{array}{l}\text { Cells are not adjusted to } \\
\text { grow on glass conditions }\end{array}$} & $\begin{array}{l}\text { Adjust cells to glass growth conditions prior to } \\
\text { starting an immunofluorescence experiment by } \\
\text { performing a few passages on coverslips prior to } \\
\text { starting an experiment }\end{array}$ \\
\hline & & & $\begin{array}{l}\text { Dilute cells in the final growth medium volume } \\
\text { prior to seeding to ensure even spreading } \\
\text { across the coverslip }\end{array}$ \\
\hline 3 & $\begin{array}{l}\text { Inefficient antibody } \\
\text { labeling }\end{array}$ & $\begin{array}{l}\text { Epitopes are } \\
\text { inaccessible for } \\
\text { antibodies with standard } \\
\text { fixation }\end{array}$ & $\begin{array}{l}\text { Pre-fixation with } 2 \% \text { formaldehyde, then fixation } \\
\text { with ice-cold methanol for } 5 \text { min }\end{array}$ \\
\hline \multirow[t]{2}{*}{$\begin{array}{l}11 \\
\text { and } \\
14\end{array}$} & \multirow[t]{2}{*}{ High background signal } & $\begin{array}{l}\text { Antibody concentration } \\
\text { was too high or washing } \\
\text { was performed } \\
\text { improperly }\end{array}$ & $\begin{array}{l}\text { Reduce antibody concentration, increase } \\
\text { washing steps and/or PBST volume; wash } \\
\text { samples thoroughly by a mild incubation on a } \\
\text { rocking platform }\end{array}$ \\
\hline & & $\begin{array}{l}\text { Antibody was not } \\
\text { appropriate for } \\
\text { immunofluorescence or } \\
\text { not reactive }\end{array}$ & Use an alternative antibody \\
\hline \multirow[t]{2}{*}{$\begin{array}{l}11 \\
\text { and } \\
14\end{array}$} & $\begin{array}{l}\text { High background signal } \\
\text { and/or abnormally } \\
\text { wrinkled nuclear shape }\end{array}$ & Sample dried out & $\begin{array}{l}\text { Make sure the stepwise exchange of fixative is } \\
\text { followed to avoid sample drying out }\end{array}$ \\
\hline & $\begin{array}{l}\text { High background signal } \\
\text { combined with little or no } \\
\text { specific signal }\end{array}$ & $\begin{array}{l}\text { Antibody aggregates } \\
\text { have formed }\end{array}$ & $\begin{array}{l}\text { If antibody have aggregated their affinity can } \\
\text { vary. Unless absolutely required, we do not } \\
\text { recommend overnight incubations, in order to } \\
\text { avoid high background signal. Keep antibodies } \\
\text { at } 4{ }^{\circ} \mathrm{C} \text { after thawing, or do not freeze antibodies } \\
\text { that do not contain cryopreservants }\end{array}$ \\
\hline \multirow[t]{6}{*}{25} & Coverslip instability & $\begin{array}{l}\text { Excess of Vectashield } \\
\text { around coverslip leads } \\
\text { to a compromised } \\
\text { sealant polymerization }\end{array}$ & $\begin{array}{l}\text { Effectively remove excess mounting medium by } \\
\text { blot-drying Vectashield with a soft tissue across } \\
\text { the periphery of the coverslip. Avoid pressing } \\
\text { against the coverslip. Apply sealant generously } \\
\text { with a thin brush kept at a vertical angle towards } \\
\text { the coverslip. }\end{array}$ \\
\hline & $\begin{array}{l}\text { Precipitates in one } \\
\text { channel }\end{array}$ & $\begin{array}{l}\text { Secondary antibody has } \\
\text { precipitates }\end{array}$ & $\begin{array}{l}\text { Centrifuge antibody at } 12,000 \mathrm{~g} \text { for } 30 \mathrm{~min} \text { at } \\
4^{\circ} \mathrm{C} \text {. Use the supernatant. Alternatively, filter } \\
\text { the antibody solution through a } 0.2 \mu \mathrm{m} \text { filter }\end{array}$ \\
\hline & $\begin{array}{l}\text { Autofluorescent } \\
\text { precipitates in all } \\
\text { channels }\end{array}$ & $\begin{array}{l}\text { PBS or other solutions } \\
\text { have precipitates }\end{array}$ & Filter all solution with a $0.4 \mu \mathrm{m}$ filter \\
\hline & $\begin{array}{l}\text { Moving fluorescent } \\
\text { particles }\end{array}$ & $\begin{array}{l}\text { Secondary antibodies } \\
\text { were too old or were not } \\
\text { fixed or washed off } \\
\text { properly; or postfixation } \\
\text { step was omitted }\end{array}$ & $\begin{array}{l}\text { Use a fresh antibody batch and ensure that the } \\
\text { post-fixation step is performed and washes are } \\
\text { followed thoroughly }\end{array}$ \\
\hline & \multirow[t]{2}{*}{ Poor antibody staining } & $\begin{array}{l}\text { Fixation method is } \\
\text { inappropriate }\end{array}$ & Test both $2 \%$ and $4 \%$ formaldehyde fixation \\
\hline & & $\begin{array}{l}\text { Epitopes can be masked } \\
\text { and thus be inaccessible } \\
\text { to the antibodies. Few } \\
\text { antibodies may require } \\
\text { methanol treatment for } \\
\text { epitope accessibility }\end{array}$ & $\begin{array}{l}\text { Alternatively, formaldehyde-fixed sample can be } \\
\text { treated with } 100 \% \text { ice-cold methanol for } \\
5-10 \text { min, followed by stepwise exchange to } \\
\text { washing buffer }\end{array}$ \\
\hline
\end{tabular}


TABLE 2 | Troubleshooting table (continued).

\begin{tabular}{|c|c|c|c|}
\hline Step & Problem & Possible reason & Solution \\
\hline & Reconstruction artifacts & $\begin{array}{l}\text { Several reconstruction } \\
\text { artifacts based on } \\
\text { photobleaching or other } \\
\text { sample parameters }\end{array}$ & $\begin{array}{l}\text { For a detailed guide on assessing sample } \\
\text { reliability and presence of artifacts, see ref. } \\
29,34\end{array}$ \\
\hline & & $\begin{array}{l}\text { Slide was not properly } \\
\text { fitted to the microscope }\end{array}$ & $\begin{array}{l}\text { Clean edges of slide with a dry tissue and } \\
\text { readjust slide onto the stage holder }\end{array}$ \\
\hline & & stage & $\begin{array}{l}\text { If frosted slides are used, mount coverslip on the } \\
\text { non-frosted side to avoid tilt }\end{array}$ \\
\hline \multirow[t]{2}{*}{35} & $\begin{array}{l}\text { Segmentation of nucleus } \\
\text { is not accurate }\end{array}$ & $\begin{array}{l}\text { Fore-/background } \\
\text { calculation cannot } \\
\text { discriminate features } \\
\text { based on the pixel } \\
\text { intensities of image }\end{array}$ & $\begin{array}{l}\text { Choose a different segmentation } \\
\text { procedure/Segmenter algorithm in TANGO, e.g., } \\
\text { Huang or Otsu }\end{array}$ \\
\hline & $\begin{array}{l}\text { Nuclear edge is not well } \\
\text { recognized for } \\
\text { segmentation }\end{array}$ & $\begin{array}{l}\text { Depending on cell type, } \\
\text { the nuclear boundary is } \\
\text { more or less } \\
\text { pronounced. The } \\
\text { nuclear edge in stem } \\
\text { cells is not as sharp as } \\
\text { in somatic cells }\end{array}$ & $\begin{array}{l}\text { Use a different segmentation routine, e.g., } \\
\text { 'Hysteresis segmenter' }\end{array}$ \\
\hline 38 & $\begin{array}{l}\text { Holes and invaginations } \\
\text { in mask }\end{array}$ & $\begin{array}{l}\text { Segmentation procedure } \\
\text { was not optimal to } \\
\text { detect stained chromatin } \\
\text { as continuous region }\end{array}$ & $\begin{array}{l}\text { Apply several rounds of morphological } \\
\text { postfiltering including 'Fill holes' option until a } \\
\text { continuous volume is masked }\end{array}$ \\
\hline 42 & $\begin{array}{l}\text { Problems with setup of } \\
\text { measurements in } \\
\text { TANGO }\end{array}$ & $\begin{array}{l}\text { Suboptimal selection of } \\
\text { measurement } \\
\text { parameters }\end{array}$ & $\begin{array}{l}\text { Check the TANGO online-site for help } \\
\text { (http://biophysique.mnhn.fr/tango/HomePage) }\end{array}$ \\
\hline \multirow[t]{2}{*}{45} & $\begin{array}{l}\text { Segmentation of objects } \\
\text { is not accurate }\end{array}$ & $\begin{array}{l}\text { Object seeds are not } \\
\text { prominent enough for } \\
\text { watershed-based } \\
\text { segmentation }\end{array}$ & $\begin{array}{l}\text { Apply pre-filters emphasizing spot edges from } \\
\text { background (TopHat, LoG) }\end{array}$ \\
\hline & & $\begin{array}{l}\text { Signal-to-noise is not } \\
\text { sufficient for edge } \\
\text { detection }\end{array}$ & Adjust post-filtering (SNR-ratio and so on) \\
\hline 47 & $\begin{array}{l}\text { Algorithm cannot } \\
\text { separate image features } \\
\text { into back- and foreground }\end{array}$ & $\begin{array}{l}\text { Poor choice of threshold } \\
\text { algorithm }\end{array}$ & $\begin{array}{l}\text { As standard, Otsu, Moments and Huang should } \\
\text { be chosen. In rare cases, stricter algorithms, } \\
\text { such as MaxEntropy or Intermodes, can be } \\
\text { applied }\end{array}$ \\
\hline
\end{tabular}

\section{ANTICIPATED RESULTS}

The presented protocol offers the possibility to perform high-throughput analyses of single-cell information extracted from 3D-SIM data sets. Following the described workflows, the user is able to obtain quantitative measurements of macromolecular spatial relationships of nuclear structures, epigenetic marks and factors, and correlate their distributions to validated standards as previously shown ${ }^{26,27}$.

Key statistics of mammalian replication foci can be extracted from high-resolution 3D-SIM data, leading to a 3-fold increase in replication foci counts compared to the numbers observed with conventional widefield deconvolution data (Fig. 3c). The use of automated analysis platforms (Fig. 3; Supplementary Fig. 2) offers convenience and unbiased analysis and allows system calibration checks in in vitro as well as biological calibration samples as reference ruler standards for biological follow-up in vivo experiments (Fig. 4). 
Minimal distances can be calculated with high accuracy between nearest neighbor foci-pairs from centroid localization of segmented structures (Supplementary Table 2).

Through the application of these analysis pipelines we show a significant spatial correlation of early-replicating chromatin to histone modifications that are linked to early $S$ phase, compared to a much lower correlation to late replicating chromatin. Finally, we are able to confirm the spatial difference between epigenetic marks that are known to occur in disparate chromatin states (Fig. 5; Supplementary Table 2). These applications demonstrate that close or distant localizations of nuclear structures or epigenetic factors can not only be observed in immunofluorescence images, but now can be measured bioinformatically at the nanometer resolution. This protocol can, therefore, serve as a versatile tool to compare distributions and correlations of nuclear structures and epigenetic factors across different cell types and states that will enable researchers to further elucidate principles that dictate spatial genome organization and function.

\section{ACKNOWLEDGEMENTS}

We thank Philip Tinnefeld and Mario Raab (TU Braunschweig, Germany) for the development and assistance with the preparation and image acquisition of DNA-origami rods. This work was funded by the Deutsche Forschungsgemeinschaft (SFB 1064 and Nanosystems Initiative Munich, NIM) and the Wellcome Trust Strategic Awards 091911 and 107457 supporting advanced microscopy at Micron Oxford. J.D. is supported by the NIHOxford-Cambridge Scholars Program. A.M. is supported by JSPS KAKENHI Grant Numbers JP16H01440 ('resonance bio'), JP15K14500 and JP26292169.

\section{AUTHOR CONTRIBUTIONS}

F.K., E.M., A.B., T.C. and Q.A. performed the experiments. F.K. established the image analysis workflows. J.D., F.K., E.M., Y.M. and L.S. wrote the manuscript. A.M. established image registration procedures. Y.M., L.S., and F.K. directed experiments and designed the protocol. H.L., Y.M., and L.S. conceived the project.

\section{COMPETING FINANCIAL INTERESTS}

The authors declare no competing financial interest. 


\section{REFERENCES}

1 Hell, S. W. Far-field optical nanoscopy. Science 316, 1153-1158 (2017).

2 Schermelleh, L., Heintzmann, R. \& Leonhardt, H. A guide to super-resolution fluorescence microscopy. J Cell Biol 190, 165-175 (2010).

3 Demmerle, J., Wegel, E., Schermelleh, L. \& Dobbie, I. M. Assessing resolution in super-resolution imaging. Methods 88, 3-10 (2015).

4 Huang, B., Babcock, H. \& Zhuang, X. Breaking the diffraction barrier: superresolution imaging of cells. Cell 143, 1047-1058 (2010).

5 Liu, Z., Lavis, L. D. \& Betzig, E. Imaging live-cell dynamics and structure at the single-molecule level. Mol Cell 58, 644-659 (2015).

6 Gustafsson, M. G. Surpassing the lateral resolution limit by a factor of two using structured illumination microscopy. J Microsc 198, 82-87 (2000).

7 Heintzmann, R. \& Cremer, C. Laterally modulated excitation microscopy: Improvement of resolution by using a diffraction grating. $P$ Soc Photo-Opt Ins $\mathbf{3 5 6 8 ,}$ 185-196 (1999).

8 Gustafsson, M. G. et al. Three-dimensional resolution doubling in wide-field fluorescence microscopy by structured illumination. Biophys J 94, 4957-4970 (2018).

9 Schermelleh, L. et al. Subdiffraction multicolor imaging of the nuclear periphery with 3D structured illumination microscopy. Science 320, 1332-1336 (2008).

10 Fornasiero, E. F. \& Opazo, F. Super-resolution imaging for cell biologists: concepts, applications, current challenges and developments. Bioessays 37, 436-451 (2015).

11 Lakadamyali, M. \& Cosma, M. P. Advanced microscopy methods for visualizing chromatin structure. FEBS Lett 589, 3023-3030 (2015).

12 Gao, J. Y. X. N., M.; Yang, D.; Xi, W.P.; Zhang, M.Q. Developing bioimaging and quantitative methods to study 3D genome. Quantitative Biology 4, 129-147 (2016).

13 Wegel, E. et al. Imaging cellular structures in super-resolution with SIM, STED and Localisation Microscopy: A practical comparison. Sci Rep 6, 27290 (2016).

14 Ricci, M. A., Manzo, C., Garcia-Parajo, M. F., Lakadamyali, M. \& Cosma, M. P. Chromatin fibers are formed by heterogeneous groups of nucleosomes in vivo. Cell 160, 1145-1158 (2015).

15 Boettiger, A. N. et al. Super-resolution imaging reveals distinct chromatin folding for different epigenetic states. Nature 529, 418-422 (2016).

16 Fraser, J., Williamson, I., Bickmore, W. A. \& Dostie, J. An Overview of Genome Organization and How We Got There: from FISH to Hi-C. Microbiol Mol Biol Rev 79, 347-372 (2015).

17 Nora, E. P. et al. Spatial partitioning of the regulatory landscape of the X-inactivation centre. Nature 485, 381-385 (2012).

18 Dekker, J. Mapping the 3D genome: Aiming for consilience. Nat Rev Mol Cell Biol 17, 741-742 (2016).

19 Fabre, P. J. et al. Nanoscale spatial organization of the HoxD gene cluster in distinct transcriptional states. Proc Natl Acad Sci U S A 112, 13964-13969 (2015).

20 Shivanandan, A., Radenovic, A. \& Sbalzarini, I. F. MosaiclA: an ImageJ/Fiji plugin for spatial pattern and interaction analysis. BMC Bioinformatics 14, 349 (2013).

21 Collins, T. J. Image J for microscopy. Biotechniques 43, 25-30 (2007).

22 Schneider, C. A., Rasband, W. S. \& Eliceiri, K. W. NIH Image to ImageJ: 25 years of image analysis. Nat Methods 9, 671-675 (2012).

23 Carpenter, A. E. et al. CellProfiler: image analysis software for identifying and quantifying cell phenotypes. Genome Biol 7, R100 (2006).

24 Soliman, K. CellProfiler: Novel Automated Image Segmentation Procedure for SuperResolution Microscopy. Biol Proced Online 17, 11 (2015).

25 Ollion, J., Cochennec, J., Loll, F., Escude, C. \& Boudier, T. TANGO: a generic tool for high-throughput 3D image analysis for studying nuclear organization.

Bioinformatics 29, 1840-1841 (2013).

26 Cerase, A. et al. Spatial separation of Xist RNA and polycomb proteins revealed by superresolution microscopy. Proc Natl Acad Sci U S A 111, 2235-2240 (2014). 
27 Smeets, D. et al. Three-dimensional super-resolution microscopy of the inactive $X$ chromosome territory reveals a collapse of its active nuclear compartment harboring distinct Xist RNA foci. Epigenetics Chromatin 7, 8 (2014).

28 Schmied, J. J. et al. DNA origami nanopillars as standards for three-dimensional superresolution microscopy. Nano Lett 13, 781-785 (2013).

29 Demmerle, J. et al. Strategic and practical guidelines for successful structured illumination microscopy. Nat. Protoc 12, 1011-1028 (2017).

30 Cox, S. Super-resolution imaging in live cells. Dev Biol 401, 175-181 (2015).

31 Horn, H. F. et al. A mammalian KASH domain protein coupling meiotic chromosomes to the cytoskeleton. J Cell Biol 202, 1023-1039 (2013).

32 Jennings, P. C., Cox, G. C., Monahan, L. G. \& Harry, E. J. Super-resolution imaging of the bacterial cytokinetic protein FtsZ. Micron 42, 336-341 (2011).

33 Komis, G. et al. Superresolution live imaging of plant cells using structured illumination microscopy. Nat Protoc 10, 1248-1263 (2015).

34 Ball, G. et al. SIMcheck: a Toolbox for Successful Super-resolution Structured Illumination Microscopy. Sci Rep 5, 15915 (2015).

35 Shao, L., Kner, P., Rego, E. H. \& Gustafsson, M. G. Super-resolution 3D microscopy of live whole cells using structured illumination. Nat Methods 8, 1044-1046 (2011).

36 Lesterlin, C., Ball, G., Schermelleh, L. \& Sherratt, D. J. RecA bundles mediate homology pairing between distant sisters during DNA break repair. Nature 506, 249 253 (2014).

37 Kner, P., Chhun, B. B., Griffis, E. R., Winoto, L. \& Gustafsson, M. G. Superresolution video microscopy of live cells by structured illumination. Nat Methods $\mathbf{6}$, 339-342 (2009).

38 Schermelleh, L. et al. Trapped in action: direct visualization of DNA methyltransferase activity in living cells. Nat Methods 2, 751-756 (2005).

39 Baddeley, D. et al. Measurement of replication structures at the nanometer scale using super-resolution light microscopy. Nucleic Acids Res 38, e8 (2010).

40 Chagin, V. O. et al. 4D Visualization of replication foci in mammalian cells corresponding to individual replicons. Nat Commun 7, 11231 (2016).

41 Bratton, B. P. \& Shaevitz, J. W. Simple Experimental Methods for Determining the Apparent Focal Shift in a Microscope System. PLoS One 10, e0134616 (2015).

42 Krizek, P., Lukes, T., Ovesny, M., Fliegel, K. \& Hagen, G. M. SIMToolbox: a MATLAB toolbox for structured illumination fluorescence microscopy. Bioinformatics 32, 318-320 (2016).

43 MacDonald, L., Baldini, G. \& Storrie, B. Does super-resolution fluorescence microscopy obsolete previous microscopic approaches to protein co-localization? Methods Mol Biol 1270, 255-275 (2015).

44 Haris, K., Efstratiadis, S. N., Maglaveras, N. \& Katsaggelos, A. K. Hybrid image segmentation using watersheds and fast region merging. leee $T$ Image Process 7 , 1684-1699 (1998).

45 Schindelin, J. et al. Fiji: an open-source platform for biological-image analysis. Nat Methods 9, 676-682 (2012).

46 Linkert, M. et al. Metadata matters: access to image data in the real world. $J$ Cell Biol 189, 777-782 (2010).

47 Young, L. J., Strohl, F. \& Kaminski, C. F. A Guide to Structured Illumination TIRF Microscopy at High Speed with Multiple Colors. J Vis Exp, doi:10.3791/53988 (2016).

48 Muller, M., Monkemoller, V., Hennig, S., Hubner, W. \& Huser, T. Open-source image reconstruction of super-resolution structured illumination microscopy data in ImageJ. Nat Commun 7, 10980 (2016).

49 Spitzer, M., Wildenhain, J., Rappsilber, J. \& Tyers, M. BoxPlotR: a web tool for generation of box plots. Nat Methods 11, 121-122 (2014). 


\section{FIGURES}

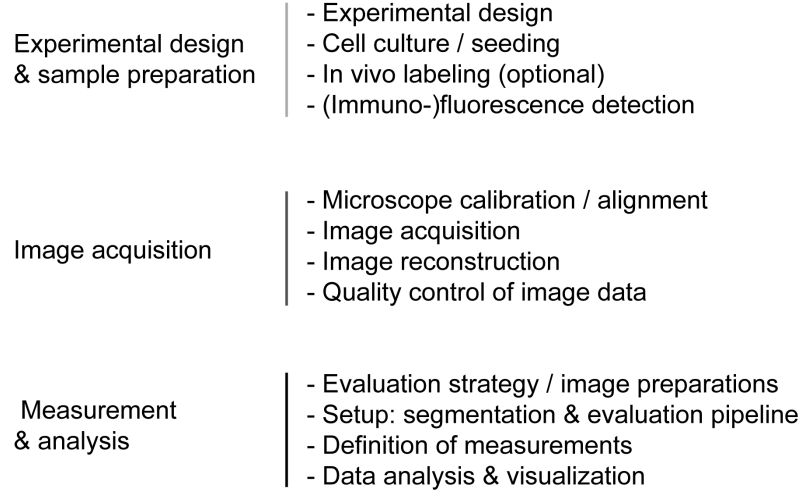

Figure 1 | Flowchart of the experimental workflow.

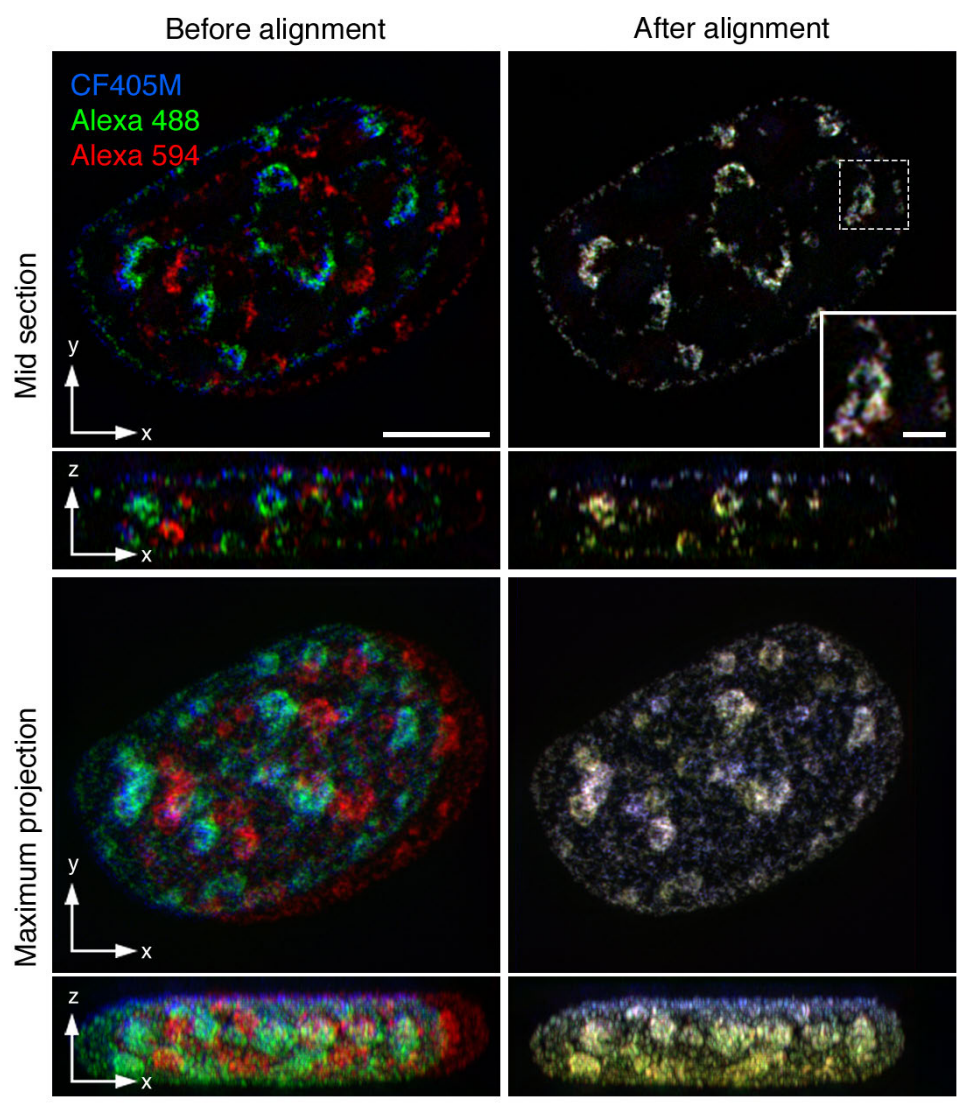

Figure 2 | Biological calibration sample for 3D color channel alignment. Super-resolution image of 3-color EdU pulse labeled $(10 \mathrm{~min})$ mouse $\mathrm{C} 127$ cell in mid-late $\mathrm{S}$ phase stage before (left column) and after (right column) 3D image registration. Incorporated EdU was detected by a simultaneous 3-color click reaction at a 7:2:1 ratio of azide-tagged CF405M (blue), Alexa Fluor 594 (red) and Alexa Fluor 488 (green), respectively. Note the enhanced bleaching of the blue CF405 dye during data acquisition as shown by the color gradient from top (white) to bottom (yellow) in the axial view of the maximum projection. Scale bars, $5 \mu \mathrm{m}$ and $0.5 \mu \mathrm{m}$ (inset). 

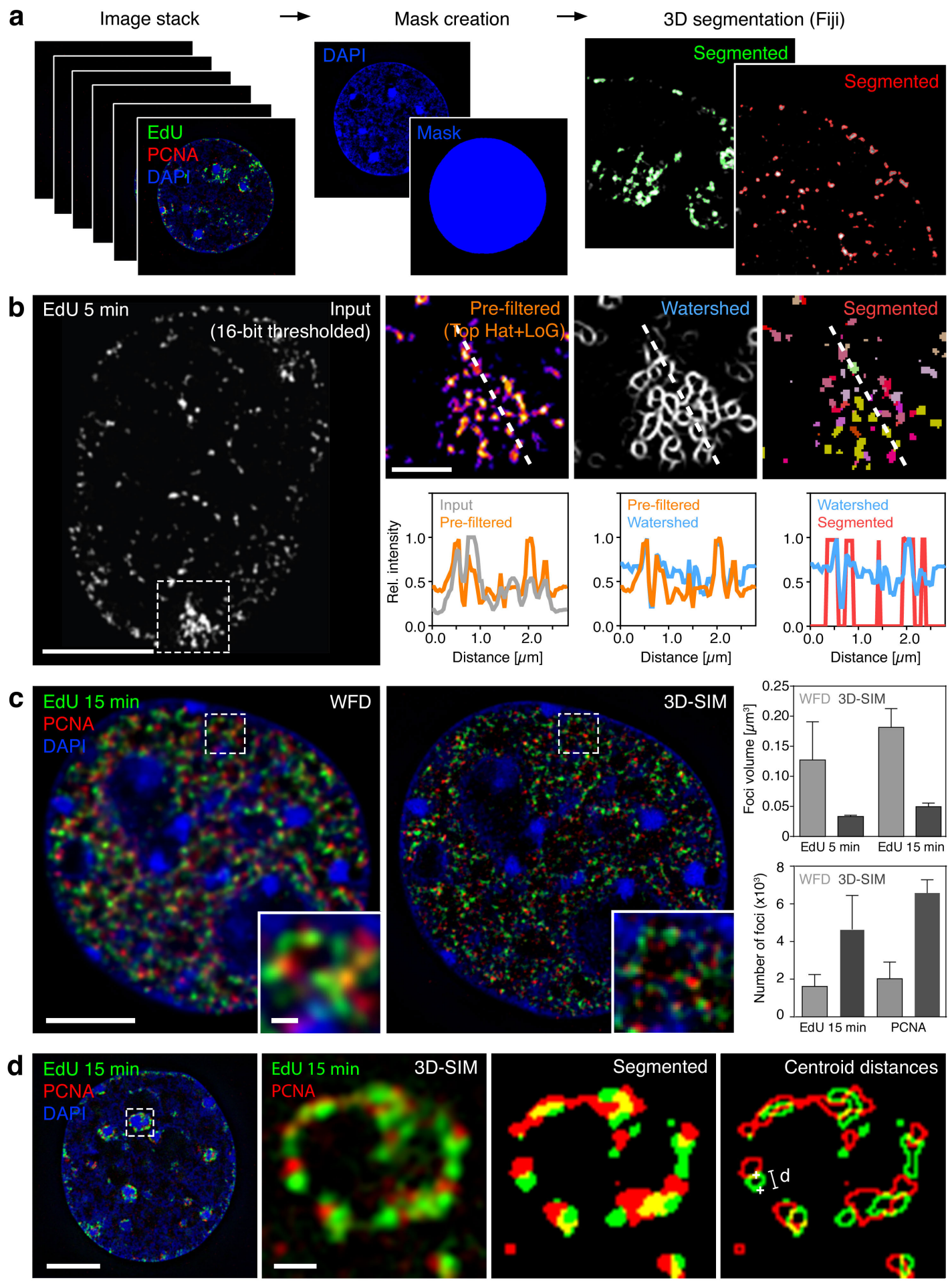

Figure 3 | Outline of image segmentation procedure. (a) Pulse EdU- and PCNA-labeled C127 cell. The region of interest $(\mathrm{ROI})$ is limited to the nuclear volume by generating a 3D mask from the DAPI counterstain. Features within the ROI are considered for the subsequent volumetric analysis. Segmentation of EdU (green) and PCNA (red) signals using built-in tools in Fiji is represented in the overlay of the input and segmented signals. (b) Example of EdU foci segmentation with TANGO. Input image (left) and magnification of replicating nuclear region prefiltered for contrast enhancement using TopHat and Laplace-ofGaussian (LoG) kernels followed by 3D seed segmentation based on a watershed algorithm. 
The steepest slope between local minima and maxima are determined and used for the 3D rendering. Plots from left to right: postfiltering of raw-segmentation image by signal-to-noise ratio (SNR) and size-exclusion filters ensures high accuracy and reduces false-positive signals. Final segmentation result (right) of the replicating nuclear region, showing the precise segmentation of even dense signal clusters. Scale bars, 5 and $1 \mu \mathrm{m}$ (inset). (c) C127 cells pulsed with EdU for 15 min and immunolabeled for PCNA. Chromatin was counterstained with DAPI. A wide-field image stack from projected raw data was deconvolved (wide-field deconvolution (WFD)) and compared with the reconstructed superresolution image from the same raw data (3D-SIM). Scale bars, 5 and $0.5 \mu \mathrm{m}$ (inset). Bottom (left panel): quantification of foci volume from segmented EdU or PCNA foci acquired with WFD or 3D-SIM data. Bottom (right panel) panel: quantification of replication foci number from segmented EdU or PCNA foci acquired with WFD or 3D-SIM data, demonstrating the superior spatial separation of complex and volumetrically dense structures with 3D-SIM. Error bars indicate standard deviations ( $n=12$ cells). (d) Example of calculation of minimal distances between segmented objects from two differently labeled structures (EdU, PCNA). In the original image (left) and inset (second from left), objects are segmented and the centroid nearest neighbors (NN) are annotated (second from right), where pairs of segmented objects in nearest proximity are determined and their spatial distance $(d)$ is computed in 3D (right). Scale bars, 5 and $0.5 \mu \mathrm{m}$ (inset). PCNA, proliferating cell nuclear antigen. 

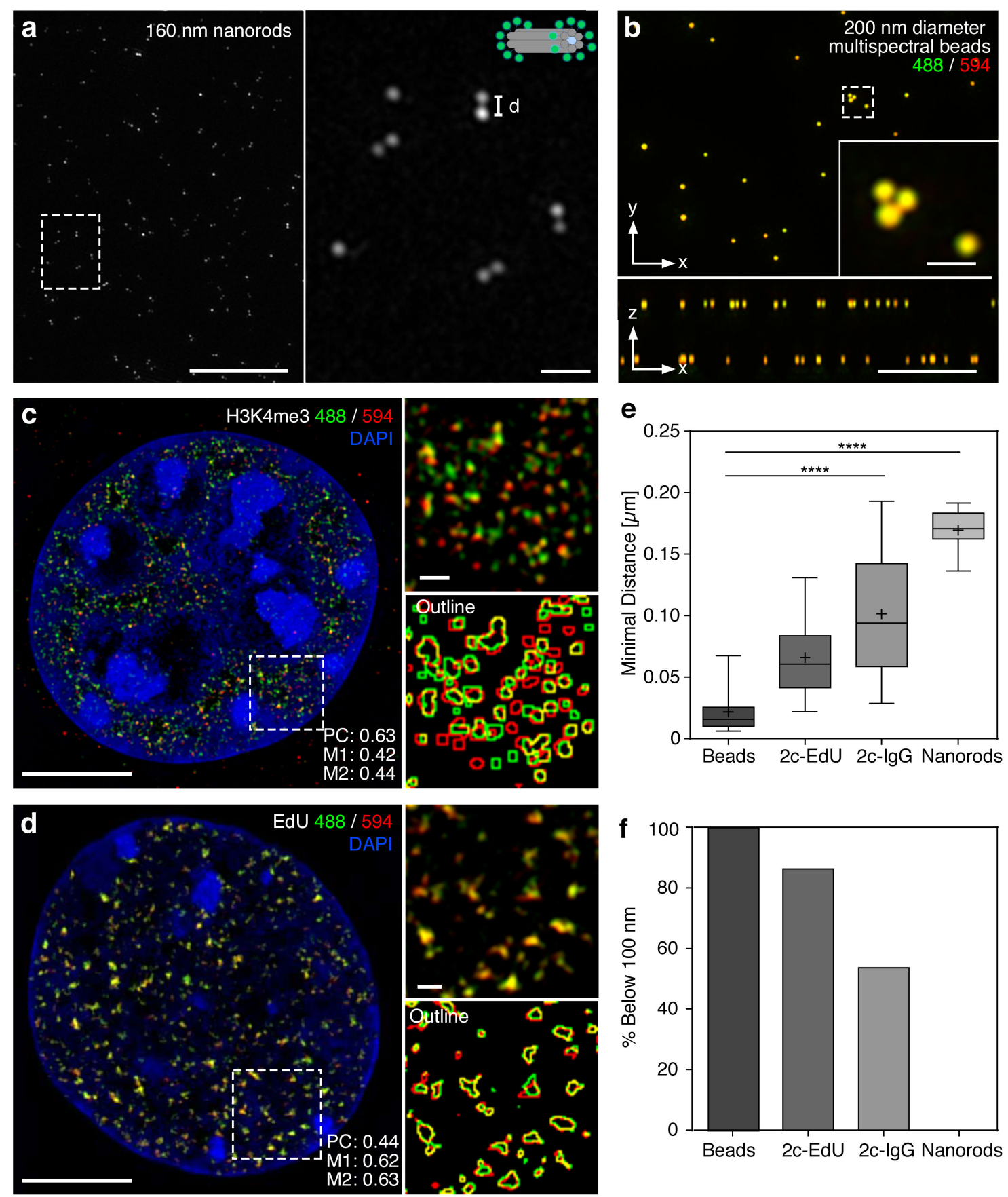

Figure 4 | Defining proximity values with in vitro and biological calibration samples imaged by 3D-SIM. (a) Maximum projection of 160 -nm-long DNA-origami nanorods bearing 10 ATTO 488 fluorophores on each end. For preparation of DNA origami slides, see

Supplementary Method 1. Scale bars, $5 \mu \mathrm{m}$ and $0.5 \mu \mathrm{m}$ (magnification). (b) Lateral (top) and orthogonal (bottom) projection of 200-nm multifluorescence TetraSpeck microspheres adhering to the slide (upper layer) and coverslip surface (lower layer). Scale bars, 2 and 0.5 $\mu \mathrm{m}$ (inset); $n=655$ DNA-nanorods. For preparation details of dual-layer TetraSpeck bead slides, see Demmerle et al. ${ }^{29}$. (c) Detection of one primary antibody (H3K4me3) with two differentially labeled secondary antibodies (Alexa Fluor 488 (green) and Alexa Fluor 594 (red); $n=5$ cells). Scale bars, 5 and $0.5 \mu \mathrm{m}$ (magnification). (d) Direct labeling of replicated DNA by dual-color EdU detection with Alexa Fluor 488- (green) and Alexa Fluor 594-azide (red) $(n=22$ cells). DNA is counterstained with DAPI. Scale bars, 5 and $0.5 \mu \mathrm{m}$ (magnification). (e) Boxplot of minimal distances after centroid localization based on nearest- 
neighbor (NN) analysis, with whiskers indicating standard deviation, cross indicating the mean and horizontal line indicating the median. The measured average minimal distance between nanorod ends is $169 \pm 16 \mathrm{~nm}$. The average minimal distance between the red and green channels of dual-stained microspheres (beads) is $16 \pm 10 \mathrm{~nm}$, that of dual-color directly labeled DNA (2c-EdU) is $61 \pm 27 \mathrm{~nm}$ and that of dual secondary antibody labeling $(2 \mathrm{c}-\operatorname{lgG})$ is $100 \pm 50 \mathrm{~nm}$. (f) Percentages of nearest neighbors calculated in $\mathbf{e}$ that are $<100$ nm apart.

a

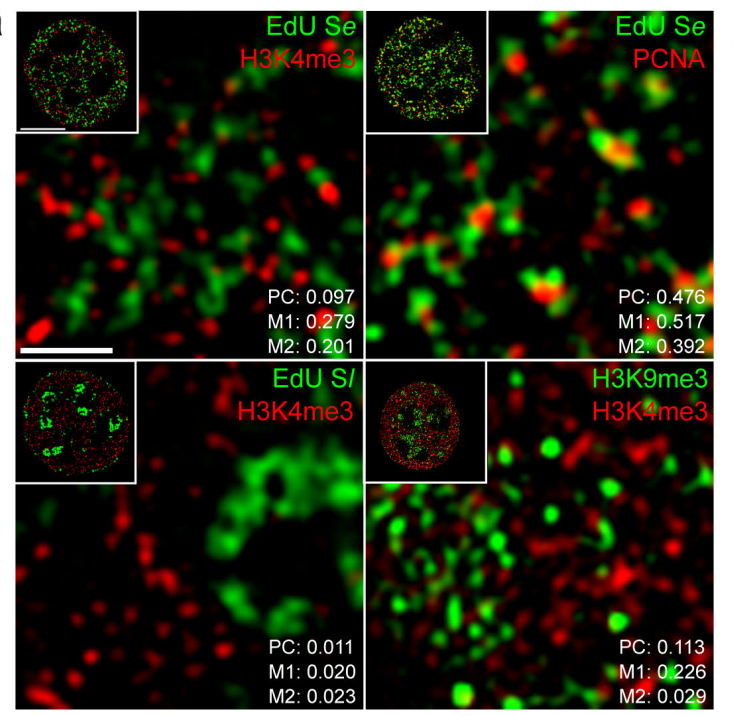

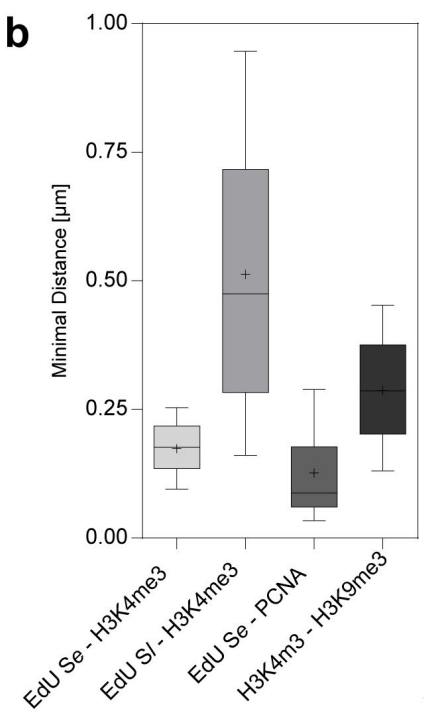

Figure 5 | Minimal distance and correlation measurements of epigenetic marks and replicating chromatin. (a) $5 \times$ magnified regions of $\mathrm{C} 127$ nucleus (insets) pulsed for 15 min with EdU (green) and immunolabeled with $\mathrm{H} 3 \mathrm{~K} 4 \mathrm{me} 3$ or PCNA antibodies (red) or dual-immunolabeled with typical euchromatin (H3K4me3, red) and heterochromatin marks (H3K9me3, green). Insets: optical sections of the corresponding nuclei. Top panels depict characteristic patterns of early (Se) and bottom left panel of late (SI) S phase replicating nuclei. Average Pearson's (PC) and Mander's (M1 and M2) correlation coefficients are indicated. Scale bars, 1 and $5 \mu \mathrm{m}$ (inset). (b) In all panels, the three left categories (under the line) indicate correlation (with) or distance (to) metrics of replication site (EdU Se or EdU SI) or epigenetic mark (H3K9me3) foci relative to $\mathrm{H} 3 \mathrm{~K} 4 \mathrm{me} 3 \mathrm{foci}$, whereas the rightmost category indicates the same metrics relative to PCNA foci. Top left panel, Pearson's coefficient of cross-channel correlation. Early-replicating chromatin exhibits high similarity to $\mathrm{H} 3 \mathrm{~K} 4 \mathrm{me} 3$, indicated by higher correlation scores as compared with a clear anticorrelation to late-replicating chromatin ( $\mathrm{PC}=0.138$ and 0.016 , respectively). PCNA and replicating DNA (EdU) exhibit a more pronounced association as compared with that between euchromatin (H3K4me3) and heterochromatin (H3K9me3) $(0.375$ and 0.145 , respectively). Top right panel, NN annotation and calculation of minimal distances between replicating chromatin (EdU) and $\mathrm{H} 3 \mathrm{~K} 4 \mathrm{me} 3$ reveals the closer proximity of early-replicating chromatin (Se) to H3K4me3 as compared with late-replicating chromatin ( $\mathrm{SI}$ ) (NN = $174 \pm 63 \mathrm{~nm}$ and $\mathrm{NN}=512 \pm 300 \mathrm{~nm}$, respectively). A closer proximity is measured between the replication machinery (PCNA) and replicating DNA (EdU) as compared with distances acquired between euchromatin and heterochromatin marks (NN = $127 \pm 103 \mathrm{~nm}$ and $\mathrm{NN}=287 \pm 124 \mathrm{~nm}$ ). Bottom left and right panels; Mander's coefficients indicating the degree of overlap between channels. Early-replicating chromatin scores overlap more with $\mathrm{H} 3 \mathrm{~K} 4 \mathrm{me} 3$ than with late-replicating chromatin (M1 $=0.239$ and $0.047, \mathrm{M} 2=0.175$ and 0.033 , respectively). Replicating DNA and PCNA show a higher overlap as compared with euchromatin (H3K4me3) and heterochromatin $(\mathrm{H} 3 \mathrm{~K} 9 \mathrm{me} 3)(\mathrm{M} 1=0.136$ and $0.064, \mathrm{M} 2=0.118-0.023$, respectively $)$. Error bars indicate standard deviations. (EdU Se-H3K4me3, $n=7$ cells; EdU SI-H3K4me3, $n=9$ cells; EdU-PCNA, $n=14$ cells; H3K4me3-H3K9me3, $n=7$ cells). 


\title{
SUPPLEMENTARY INFORMATION
}

\section{Quantitative 3D structured illumination microscopy of nuclear structures}

Felix Kraus ${ }^{1,3}$, Ezequiel Miron ${ }^{2}$, Justin Demmerle ${ }^{2}$, Tsotne Chitiashvili ${ }^{1}$, Alexei Budco ${ }^{1}$, Quentin Alle ${ }^{2}$, Atsushi Matsuda ${ }^{4,5}$, Heinrich Leonhardt ${ }^{1}$, Lothar Schermelleh ${ }^{2}$, Yolanda Markaki $^{1,6}$ *

${ }^{1}$ Department of Biology II, Center for Integrated Protein Science (CIPSM) and Center for Advanced Light Microscopy (CALM), LMU, Martinsried, 82152, Germany

${ }^{2}$ Micron Advanced Bioimaging Unit, Department of Biochemistry, University of Oxford, Oxford OX1 3QU, United Kingdom

${ }^{3}$ Current address: Department of Biochemistry and Molecular Biology, Monash Biomedicine Discovery Institute, 3800, Melbourne, Australia

${ }^{4}$ Advanced ICT Research Institute Kobe, National Institute of Information and Communications Technology, Kobe 651-2492, Japan

${ }^{5}$ Graduate School of Frontier Biosciences, Osaka University, Osaka 565-0871, Japan

${ }^{6}$ Current address: David Geffen School of Medicine, Department of Biological Chemistry, UCLA, Los Angeles, CA 90095, USA

*Correspondence should be addressed to Y.M. (gmarkaki@mednet.ucla.edu) or L.S. (lothar.schermelleh@bioch.ox.ac.uk)

\section{Supplementary Code Information Supplementary Methods 1 and 2 Supplementary Tables 1-3 \\ Supplementary Figures 1-3}

\author{
Supplementary Code \\ TANGO Processing Chains \\ We provide basic processing chains for the TANGO suite for batch-oriented image \\ processing and analysis of 3D-SIM data sets (Supplementary Table 3). These comprise of \\ example processing chains for nuclear mask segmentation as well as structure \\ segmentation templates for focal and broad spots. First, the processing chains \\ 'nucleus.bson' and 'channel.bson' are imported together with their metadata files to TANGO \\ via clicking on the 'Import Processing Chains' button in the 'Connect' tab of TANGO. After \\ importing to TANGO, a variety of processing chains can be used for basic image \\ segmentation. For segmentation of the nuclear volume, the 'Nuclear_Mask' processing \\ chain can be used for nuclear stains with a prominent nuclear rim. Segmentation of less \\ prominent nuclear borders, such as stem cell nuclear staining, can be achieved with the \\ 'ESC_Mask' processing chain. Both will create the mask based on the nuclear stain, which \\ will be used to define the volume for subsequent image analysis. Further, we included two \\ more processing chain templates for segmentation of nuclear structures with either focal \\ distributions, such as short EdU-pulses ('Focal_Spot') or broader signals, such as some \\ dense antibody-labeled histone marks ('Volumetric_Spot'). After image segmentation the \\ derived objects can be analyzed. Measurements are defined in the 'Edit Experiment' > \\ 'Measurements' tab in TANGO.
}

Supplementary Code contains the Readme file as well as the processing chains and can be downloaded as a zip file. 
Supplementary Method 1| DNA nanorod preparation protocol

\section{ADDITIONAL SUPPLEMENTARY MATERIALS}

REAGENTS

- NeutrAvidin Protein (Thermo Fisher, cat. no. 31000)

- LabTek 8-well chamber (Thermo Fisher, cat. no.154534)

- Tris-EDTA buffer solution, pH 8.0 (Sigma-Aldrich, cat. no. 93283)

- $\mathrm{MgCl}_{2}$ solution (Sigma Aldrich, cat. no. M1028)

- Sodium Chloride solution (Sigma Aldrich, cat. no. 71386)

- $99 \%$ w/vol Glycerol (Sigma Aldrich, cat. no. G5516)

- Amicon Ultra-0.5 ml Centrifugal Filters (Merck Millipore, cat. no. UFC500324)

- Albumin, biotin labeled bovine, lyophilized powder (Sigma Aldrich, cat. no. A8549)

\section{REAGENT SETUP}

- Nanoruler GATTA-SIM (GATTAquant, cat. no. SIM 160B). Store at $-20^{\circ} \mathrm{C}$.

- Filtering buffer (Tris-EDTA pH 8, $12 \mathrm{mM} \mathrm{MgCl}$ ). Add $120 \mu \mathrm{IgCl}_{2}$ solution to $10 \mathrm{ml}$ Tris-EDTA buffer solution and mix by brief vortexing. Store at $4{ }^{\circ} \mathrm{C}$.

- Labeling buffer (Tris-EDTA pH 8, $50 \mathrm{mM} \mathrm{NaCl}, 12 \mathrm{mM} \mathrm{MgCl} 2$ ). Add $100 \mu \mathrm{NaCl}$ solution to $10 \mathrm{ml}$ filtering buffer and mix by brief vortexing. Store at $4{ }^{\circ} \mathrm{C}$.

- BSA-biotin solution ( $0.5 \mathrm{mg} / \mathrm{ml}$ in PBS). Add $5 \mathrm{mg}$ of albumin-biotin to $10 \mathrm{ml}$ of $1 \mathrm{x}$ PBS. Dissolve by vortexing, make $1 \mathrm{ml}$ aliquots and store at $-20^{\circ} \mathrm{C}$.

- NeutrAvidin solution ( $0.5 \mathrm{mg} / \mathrm{ml}$ NeutrAvidin in PBS): Add $5 \mathrm{mg}$ of NeutrAvidin to 10 $\mathrm{ml}$ of $1 \mathrm{x}$ PBS. Dissolve by vortexing, make $1 \mathrm{ml}$ aliquots and store at $-20{ }^{\circ} \mathrm{C}$.

- Imaging buffer (10-20 mM MgCl 2 in PBS). Add $100-200 \mu \mathrm{MgCl}_{2}$ solution in $10 \mathrm{ml}$ PBS to obtain imaging buffer containing $10-20 \mathrm{mM} \mathrm{MgCl}_{2}$.

\section{Preparation of DNA nanorods for system calibration TIMING $4 \mathbf{h}$}

Folding and external labeling of SIM-Rulers based on the 12-Helix-Bundle:

DNA nanorods were kindly designed and provided by the Tinnefeld lab as previously published ${ }^{1}$. Alternatively, nanorods can be purchased from GATTAquant.

For 3D-SIM imaging the protocol was adjusted as described below.

1) Treat DNA origami tube in a PCR-machine with this program for DNA origami folding:

$80^{\circ} \mathrm{C}, 15 \mathrm{~min}$

$80-66{ }^{\circ} \mathrm{C}, 5 \mathrm{~min} /{ }^{\circ} \mathrm{C}$

$65-30{ }^{\circ} \mathrm{C}, 30 \mathrm{~min} /{ }^{\circ} \mathrm{C}$

Hold at $4{ }^{\circ} \mathrm{C}$

PAUSE POINT product can be stored at $4{ }^{\circ} \mathrm{C}$ overnight

2| Put a centrifugal filter into an affiliated tube.

3| Fill $400 \mu$ f filtering buffer into the filter.

4| Pipette $100 \mu \mathrm{l}$ of the folded DNA origami solution into the $400 \mu \mathrm{l}$ filtering buffer, and close the cap.

5| Put the centrifugal filter into a centrifuge and spin $10 \mathrm{~min}$ at $15,000 \mathrm{~g}$ at $4{ }^{\circ} \mathrm{C}$.

6| Empty the tube containing the filtering buffer together with unbound oligonucleotides.

7| Filter in total 3 times, each time filling $400 \mu$ of filtering buffer into the filter. 
8| Take the filter set out of the tube, flip it and put it into a new, unused tube.

9| Centrifuge for $2 \mathrm{~min}$ at $1,000 \mathrm{~g}$ at $4{ }^{\circ} \mathrm{C}$.

10| Dilute the filtered DNA origami in filtering buffer. Add approx. $25 \mu$ of DNA origami solution to $70 \mu \mathrm{l}$ of labeling buffer containing the complementary oligonucleotide conjugated to Alexa-488 dye (100 nM).

11| Incubate the solution for $2 \mathrm{~h}$ at $37^{\circ} \mathrm{C}$.

12| Purify the solution with centrifugal filters as described in steps 2 to 9.

Immobilization of DNA origamis for imaging on LabTek chambers OTIMING $1 \mathbf{h}$ 13| Wash the LabTek chamber 3 times with $500 \mu \mathrm{l}$ 1x PBS.

14| Incubate the LabTek chamber with $200 \mu$ of BSA-biotin solution for 5 min.

15| Remove the BSA-biotin solution and wash three times with $500 \mu \mathrm{l}$ x PBS. Make sure not to scratch with the pipette tip on the surface. Choose one corner of the LabTek chamber and always pipette at this position.

16|| Incubate the LabTek chamber with $200 \mu$ of NeutrAvidin solution for 5 min.

17| Remove the NeutrAvidin solution and wash 3 times with $500 \mu \mathrm{l} 1 \mathrm{x}$ PBS.

18| Dilute 1-3 $\mu$ of the filtered DNA origami solution with $100 \mu$ imaging buffer.

19| Incubate the LabTek chamber with diluted DNA origami solution for $5 \mathrm{~min}$.

$\triangle$ CRITICAL STEP The optimal dilution factor to reach a dense surface for imaging depends mainly on the starting concentration of the DNA origami filtrate. This concentration depends on several factors like type of purification, type of DNA origami structure, initial scaffold concentration and age of the sample. Therefore, it is recommended to start with 1 $\mu \mathrm{l}$, check the density on the microscope and if necessary increase the density. The ideal average density of DNA origami structures on the surface is $\sim 1$ per $\mu \mathrm{m}^{2}$.

20| Remove the DNA origami solution and wash the chamber 3 times with $500 \mu \mathrm{l} 1 \mathrm{x}$ PBS.

21| Fill the chamber with the respective imaging buffer matching the requirements of the intended measurement. It should contain $10-20 \mathrm{mM} \mathrm{MgCl}_{2}$.

$\triangle$ CRITICAL STEP To maintain rigidity of the 12-Helix-Bundle, addition of $\mathrm{MgCl}_{2}$ is required until immobilization and measurement. At very low $\mathrm{Mg}^{2+}$ concentrations relatively strong bending of the nanorods occurs, which leads to considerable shortening of the mean distance between origami-poles and a broad distance distribution. We recommend $\mathrm{Mg}^{2+}$ concentrations between $10 \mathrm{mM}$ and $20 \mathrm{mM}$. In this range, only small bending occurs and the mean distance between the fluorophores after imaging and centroid allocation is marginally smaller to the originally designed distance.

22| Proceed to main protocol Step 23. 
Supplementary Method 2 | Analysis of individual images with standard ImageJ/Fiji

\section{1| Generation of nuclear volume using standard ImageJ/Fiji $\bigcirc$ TIMING 5-7 min per image stack}

Using the DAPI or other DNA/chromatin counterstain channel, create a nuclear mask to confine the region of interest to the nuclear volume and reduce the number of voxels and therefore the amount of data to be analyzed (Fig. 3a). Load the composite tif-stack into Fiji and duplicate the counterstain channel.

2| Filter the image stack by using a 3D Gaussian filter (Process >> Filters >> Gaussian Blur 3D). Apply a sigma of 2 in $x y$ and $z$ for the mask generation.

$\triangle$ CRITICAL STEP Convert the duplicated stack to a binary mask (Process >> Binary >> Convert to Mask), using a thresholding function of choice; the Otsu thresholding algorithm serves well in most cases.

3| Post-filtering of the nuclear mask: Fill holes and invaginations using Dilate and Fill Holes 2D/3D function (Process >> Binary). It is possible that multiple rounds of post-filtering are necessary, depending on the signal properties.

4| Save the mask with an appropriate name (e.g., DAPI_mask).

\section{5| Definition of Measurements using standard ImageJ/Fiji $\bigcirc$ TIMING 1 min per image stack}

Select the desired parameters to be measured under Analyze >> 3D OC Options, and save them with OK. In 3D OC Options of the 3D Objects Counter, a plethora of potential calculated image parameters can be found, ranging from centroid positions, integrated density and signal intensity based measurements. In addition, image analysis can be performed with the Analyze Particles command in the Analyze menu. In comparison to TANGO, a direct nearest neighbor analysis is not possible.

\section{6| Structure Segmentation using standard ImageJ/Fiji TIMING 2 min per image stack \\ Open the structure channel and the nuclear mask file.}

$\triangle$ CRITICAL STEP Make sure both files are of the same type of image (e.g., 8-bit image stacks).

7| Duplicate the DAPI channel, which will define the region for volumetric analysis.

8| Convert the duplicated stack to a binary mask (Process >> Binary >> Convert to Mask), using a thresholding function of choice; the Otsu thresholding algorithm serves well in most cases.

9| Fill holes and invaginations using Dilate and Fill Holes 2D/3D function (Process >> Binary). Multiple rounds of post-filtering may be needed. Save the mask with an appropriate name.

10| Invert the mask and subtract it from your structure(s) of interest channel(s), using the Image Calculator tool (Process $>>$ Image Calculator). Save the corrected structure channels under an appropriate name.

$\triangle$ CRITICAL STEP The above assignment ensures that only signals inside the selected volume will be measured and should not be omitted. 
11 Analyze the defined structure channels in a sequential manner for foci using the 3D Objects Counter (Analyze >> 3D Objects Counter) (Supplementary Fig. 2a, b).

$\triangle$ CRITICAL STEP After opening the image stack, a threshold, which will define objects and further parameters, has to be defined. Adjust appropriately and carefully, reducing chances of false-positive measurements. It is recommended to orient the threshold selection according to the image stack histogram of the selected channel. After the measurements and calculations are run, a results window will open. Since in this standard method structures are only separated based on their intensities, the threshold value is pivotal. Good estimates for threshold values are for example provided by the modal value of the image histogram stack (see SIMcheck) or by iterative intensity thresholding algorithms like Otsu or Huang already implemented in ImageJ/Fiji.

12| Measurements of image structures using standard ImageJ/Fiji $\bigcirc$ TIMING 2 min per image stack

Re-evaluate the measurements performed on the image.

$\triangle$ CRITICAL STEP Reduce computing time by choosing to extract only the necessary information required from the segmented image stack.

13| Load the image into ImageJ/Fiji.

14| Run the measurements (Analyze >> 3D Objects Counter).

15| Measurements and calculations are run and a results window opens (Supplementary Fig. 2b).

$\triangle$ CRITICAL STEP It should be noted that ImageJ/Fiji do not offer the use of well-tailored segmentation processes which can be found for example in TANGO, including a plethora of signal pre- and post-filtering steps. Since standard ImageJ/Fiji segmentation methods are mostly depending on intensity thresholding, separation of structures can fail in densely stained specimens.

\section{6| Data export and saving using standard ImageJ/Fiji $\bigcirc$ TIMING 1 min per image stack}

Results of measurements from ImageJ/Fiji can be exported and saved as .txt or .csv files for further plotting and statistical analysis, using the File $>>$ Save as... dialog in ImageJ/Fiji. 
Supplementary Table 1 | Details of antibodies

\begin{tabular}{|c|c|c|c|c|c|c|}
\hline Name & Dilution & Manufacturer & $\begin{array}{l}\text { Catalog } \\
\text { number }\end{array}$ & Fixation & Refs & $\begin{array}{l}\text { Tested cell } \\
\text { types }\end{array}$ \\
\hline \multicolumn{7}{|l|}{ Primary Antibodies } \\
\hline $\begin{array}{l}\text { Mouse monoclonal histone } \\
\text { H3K27me3 primary antibody }\end{array}$ & $1: 400$ & Active Motif & 39536 & $\begin{array}{l}2 \% \text { formaldehyde, } \\
10 \mathrm{~min}, \mathrm{RT}\end{array}$ & 2,3 & $\begin{array}{l}\text { mESCs, HeLa, } \\
\text { C2C12, C127 }\end{array}$ \\
\hline $\begin{array}{l}\text { Mouse monoclonal histone } \\
\text { H3K9me3 primary antibody }\end{array}$ & $1: 700$ & $\begin{array}{l}\text { Gift from } \\
\text { Hiroshi } \\
\text { Kimura, Kyoto } \\
\text { University }\end{array}$ & - & $\begin{array}{l}2 \% \text { formaldehyde, } \\
10 \min , \mathrm{RT}\end{array}$ & 4 & $\begin{array}{l}\text { mESCs, HeLa, } \\
\text { C2C12, C127 }\end{array}$ \\
\hline $\begin{array}{l}\text { Rabbit polyclonal histone } \\
\text { H3K4me3 primary antibody }\end{array}$ & $1: 400$ & Active Motif & 39160 & $\begin{array}{l}2 \% \text { formaldehyde, } \\
10 \mathrm{~min}, \mathrm{RT}\end{array}$ & 2,3 & $\begin{array}{l}\text { mESCs, HeLa, } \\
\text { C2C12, C127 }\end{array}$ \\
\hline $\begin{array}{l}\text { Rat monoclonal PCNA primary } \\
\text { antibody }\end{array}$ & $1: 40$ & ChromoTek & 16D10 & $\begin{array}{l}2 \% \text { formaldehyde, } \\
10 \mathrm{~min}, \mathrm{RT} \text { and ice- } \\
\text { cold } 100 \% \mathrm{MeOH} \text {, } \\
5 \mathrm{~min}\end{array}$ & 5,6 & $\begin{array}{l}\text { mESCs, HeLa, } \\
\text { C2C12, C127 }\end{array}$ \\
\hline \multicolumn{7}{|l|}{ Secondary Antibodies } \\
\hline $\begin{array}{l}\text { Donkey anti-mouse secondary } \\
\text { antibody } \lg G(H+L) \text {, Alexa Fluor } \\
488 \text { conjugated }\end{array}$ & $1: 400$ & $\begin{array}{l}\text { Invitrogen } \\
\text { ThermoFisher } \\
\text { Scientific }\end{array}$ & R37114 & & $2,3,7$ & $\begin{array}{l}\text { mESCs, HeLa, } \\
\text { C2C12, C127 }\end{array}$ \\
\hline $\begin{array}{l}\text { Goat anti-mouse secondary } \\
\text { antibody } \lg G(H+L) \text {, Alexa Fluor } \\
594 \text { conjugated }\end{array}$ & $1: 400$ & $\begin{array}{l}\text { Invitrogen } \\
\text { ThermoFisher } \\
\text { Scientific }\end{array}$ & $A-11032$ & & $2,3,7$ & $\begin{array}{l}\text { mESCs, HeLa, } \\
\text { C2C12, C127 }\end{array}$ \\
\hline $\begin{array}{l}\text { Goat anti-rabbit secondary } \\
\text { antibody } \lg G(H+L) \text {, Alexa Fluor } \\
488 \text { conjugated }\end{array}$ & $1: 400$ & $\begin{array}{l}\text { Invitrogen } \\
\text { ThermoFisher } \\
\text { Scientific }\end{array}$ & $\mathrm{R} 37116$ & & $2,3,7$ & $\begin{array}{l}\text { mESCs, HeLa, } \\
\text { C2C12, C127 }\end{array}$ \\
\hline $\begin{array}{l}\text { Donkey anti-rabbit secondary } \\
\text { antibody } \lg G(H+L) \text {, Alexa Fluor } \\
594 \text { conjugated }\end{array}$ & $1: 400$ & $\begin{array}{l}\text { Invitrogen } \\
\text { ThermoFisher } \\
\text { Scientific }\end{array}$ & $\mathrm{R} 37119$ & & $2,3,7$ & $\begin{array}{l}\text { mESCs, HeLa, } \\
\text { C2C12, C127 }\end{array}$ \\
\hline $\begin{array}{l}\text { Donkey anti-rat secondary } \\
\text { antibody } \operatorname{lgG}(\mathrm{H}+\mathrm{L}) \text {, Alexa Fluor } \\
594 \text { conjugated }\end{array}$ & $1: 400$ & $\begin{array}{l}\text { Invitrogen } \\
\text { ThermoFisher } \\
\text { Scientific }\end{array}$ & A-21209 & & 5,6 & $\begin{array}{l}\text { mESCs, HeLa, } \\
\text { C2C12, C127 }\end{array}$ \\
\hline
\end{tabular}

The table includes information on fixation, dilution and species reactivity for all antibodies used in this protocol, including citations where these antibodies were previously used. 
Supplementary Table 2 | Correlation coefficients and minimal distance measurements

\begin{tabular}{|c|c|c|c|c|c|c|c|c|}
\hline $\begin{array}{l}\text { Minimal } \\
\text { distances }(\mu \mathrm{m})\end{array}$ & Beads & $\begin{array}{l}\text { DNA } \\
\text { nanorods }\end{array}$ & IgGs & Dual-EdU & $\begin{array}{l}\text { H3K4me3- } \\
\text { EdU early } \\
\text { S phase }\end{array}$ & $\begin{array}{l}\text { H3K4me3- } \\
\text { EdU late } \\
\text { S phase }\end{array}$ & $\begin{array}{l}\text { PCNA- } \\
\text { EdU early } \\
\text { S phase }\end{array}$ & $\begin{array}{l}\text { H3K4me3- } \\
\text { H3K9me3 }\end{array}$ \\
\hline $\begin{array}{l}\text { Number of } \\
\text { values }\end{array}$ & 75 & 655 & 86918 & 41681 & 60316 & 131871 & 21122 & 56939 \\
\hline Minimum & 0 & 0.109 & 0.0014 & 0 & 0 & 0 & 0 & 0 \\
\hline $25 \%$ percentile & 0.0092 & 0.1623 & 0.0582 & 0.0405 & 0.1349 & 0.2832 & 0.06044 & 0.2022 \\
\hline Median & 0.0143 & 0.1708 & 0.0930 & 0.0587 & 0.1766 & 0.4646 & 0.0876 & 0.2866 \\
\hline $75 \%$ percentile & 0.0229 & 0.1834 & 0.1388 & 0.0796 & 0.2182 & 0.7166 & 0.1773 & 0.3754 \\
\hline Maximum & 0.0395 & 0.194 & 0.2047 & 0.1335 & 0.3063 & 1.283 & 0.4637 & 0.5643 \\
\hline Mean & 0.0162 & 0.1695 & 0.0998 & 0.0613 & 0.174 & 0.5132 & 0.1272 & 0.287 \\
\hline Std. deviation & 0.0098 & 0.01617 & 0.0503 & 0.0270 & 0.0625 & 0.2993 & 0.1032 & 0.1244 \\
\hline $\begin{array}{l}\text { Std. error of } \\
\text { the mean }\end{array}$ & 0.0011 & 0.0006 & 0.0001 & 0.0001 & 0.0002 & 0.0008 & 0.0007 & 0.0005 \\
\hline $\begin{array}{l}\text { Pearson's } \\
\text { correlation }\end{array}$ & 0.919 & & 0.63 & 0.44 & 0.138 & 0.017 & 0.375 & 0.145 \\
\hline Mander's 1 & 1 & & 0.42 & 0.62 & 0.239 & 0.047 & 0.136 & 0.064 \\
\hline Mander's 2 & 1 & & 0.44 & 0.63 & 0.175 & 0.033 & 0.128 & 0.023 \\
\hline
\end{tabular}

The table includes minimal distance and correlation coefficient measurements performed in this protocol. 
Supplementary Table 3 | Settings for processing chains

\begin{tabular}{|c|c|c|c|c|c|c|c|}
\hline Name & \multicolumn{2}{|l|}{ Pre-filters } & Segmentation & \multicolumn{4}{|l|}{ Post-filters } \\
\hline \multirow{6}{*}{ 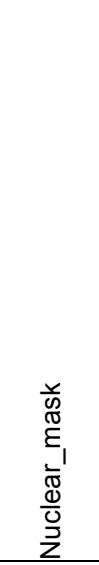 } & \multicolumn{2}{|c|}{ Misc 3D filters } & $\begin{array}{l}\text { Nucleus edge } \\
\text { detector }\end{array}$ & \multicolumn{4}{|c|}{ Morphological filters 3D } \\
\hline & \multicolumn{2}{|l|}{$\begin{array}{l}\text { Gaussian } \\
\text { 3D }\end{array}$} & $\begin{array}{l}\text { Global threshold: } \\
\text { AutoThreshold - } \\
\text { Otsu }\end{array}$ & \multirow[t]{5}{*}{ Fill holes 2D } & \multirow{5}{*}{$\begin{array}{l}\text { Binary close } \\
\text { XY-radius: } 10\end{array}$} & \multirow[t]{5}{*}{ Fill holes 2D } & \multirow{5}{*}{$\begin{array}{l}\text { Binary close } \\
\text { XY-radius: } 10\end{array}$} \\
\hline & \multirow{4}{*}{\multicolumn{2}{|c|}{ VoisXY: 2}} & $\begin{array}{l}\text { Min. nucleus size: } \\
5000\end{array}$ & & & & \\
\hline & & & Border: 5 & & & & \\
\hline & & & Gradient scale: 1 & & & & \\
\hline & & & $\begin{array}{l}\text { Local Region } \\
\text { Threshold: } \\
\text { AutoThreshold - } \\
\text { Otsu }\end{array}$ & & & & \\
\hline \multirow[b]{3}{*}{ 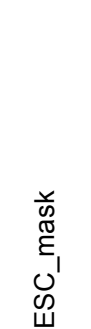 } & $\begin{array}{l}\text { Fast } \\
\text { filters 3D }\end{array}$ & $\begin{array}{l}\text { Misc 3D } \\
\text { filters }\end{array}$ & $\begin{array}{l}\text { Hysteresis } \\
\text { segmenter }\end{array}$ & \multicolumn{4}{|c|}{ Morphological Filters 3D } \\
\hline & Median & $\begin{array}{l}\text { Gaussian } \\
\text { 3D }\end{array}$ & $\begin{array}{l}\text { Threshold low: } \\
\text { AutoThreshold - } \\
\text { Otsu }\end{array}$ & \multirow[t]{2}{*}{ Fill holes 2D } & Binary close & \multirow[t]{2}{*}{ Fill holes 2D } & Binary close \\
\hline & $\begin{array}{l}\text { RadXY: } 2 \\
\text { RadZ: } 1\end{array}$ & VoisXY: 2 & $\begin{array}{l}\text { Threshold high: } \\
\text { AutoThreshold - } \\
\text { Otsu }\end{array}$ & & XY-radius: 10 & & $X Y$-radius: 10 \\
\hline \multirow{4}{*}{ 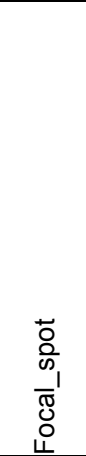 } & $\begin{array}{l}\text { Fast } \\
\text { filters 3D }\end{array}$ & $\begin{array}{l}\text { Laplacian } \\
\text { of } \\
\text { Gaussian } \\
\text { 3D }\end{array}$ & Spot detector 3D & Erase spots & $\begin{array}{l}\text { Size and edge } \\
\text { filter }\end{array}$ & & \\
\hline & TopHat & \multirow[t]{3}{*}{ VoisXY: 2} & $\begin{array}{l}\text { Background limit: } \\
\text { AutoThreshold - } \\
\text { Otsu }\end{array}$ & \multirow[t]{3}{*}{$\begin{array}{l}\text { \#1 Criterion: } \\
\text { SNR - } 2\end{array}$} & \multirow[t]{3}{*}{ Min volume: 2} & & \\
\hline & \multirow[t]{2}{*}{ RadXY: 1} & & $\begin{array}{l}\text { Seeds - Hessian } \\
\text { upper limit: } \\
\text { percentage of } \\
\text { bright pixels }(95 \%)\end{array}$ & & & & \\
\hline & & & $\begin{array}{l}\text { Seed Threshold: } \\
\text { AutoThreshold - } \\
\text { Otsu }\end{array}$ & & & & \\
\hline \multirow{4}{*}{ 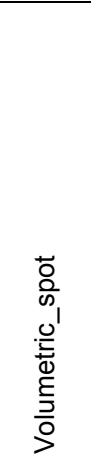 } & $\begin{array}{l}\text { Fast } \\
\text { filters 3D }\end{array}$ & $\begin{array}{l}\text { Laplacian } \\
\text { of } \\
\text { Gaussian } \\
\text { 3D }\end{array}$ & Spot detector 3D & Erase spots & $\begin{array}{l}\text { Size and edge } \\
\text { filter }\end{array}$ & & \\
\hline & TopHat & \multirow[t]{3}{*}{ Vois XY: 2} & $\begin{array}{l}\text { Background Limit: } \\
\text { AutoThreshold - } \\
\text { Otsu }\end{array}$ & \multirow[t]{3}{*}{$\begin{array}{l}\text { \#1 Criterion: } \\
\text { SNR - } 1\end{array}$} & \multirow[t]{3}{*}{ Min volume: 2} & & \\
\hline & $\operatorname{RadXY:} 2$ & & $\begin{array}{l}\text { Seeds - Hessian } \\
\text { upper limit: } \\
\text { percentage of } \\
\text { bright pixels }(95 \%)\end{array}$ & & & & \\
\hline & RadZ: 1 & & $\begin{array}{l}\text { Seed Threshold: } \\
\text { AutoThreshold - } \\
\text { Otsu }\end{array}$ & & & & \\
\hline
\end{tabular}

Table includes the processing chains parameters provided in the Supplementary Code. 


\section{SUPPLEMENTARY REFERENCES}

1 Schmied, J. J. et al. DNA origami nanopillars as standards for three-dimensional superresolution microscopy. Nano Lett 13, 781-785.

2 Cerase, A. et al. Spatial separation of Xist RNA and polycomb proteins revealed by superresolution microscopy. Proc Natl Acad Sci U S A 111, 2235-2240.

3 Smeets, D. et al. Three-dimensional super-resolution microscopy of the inactive $X$ chromosome territory reveals a collapse of its active nuclear compartment harboring distinct Xist RNA foci. Epigenetics Chromatin 7, 8.

4 Kimura, H., Hayashi-Takanaka, Y., Goto, Y., Takizawa, N. \& Nozaki, N. The organization of histone $\mathrm{H} 3$ modifications as revealed by a panel of specific monoclonal antibodies. Cell Struct Funct 33, 61-73 (2008).

5 Rottach, A. et al. Generation and characterization of a rat monoclonal antibody specific for PCNA. Hybridoma (Larchmt) 27, 91-98.

6 Schneider, K. et al. Dissection of cell cycle-dependent dynamics of Dnmt1 by FRAP and diffusion-coupled modeling. Nucleic Acids Res 41, 4860-4876.

7 Markaki, Y. et al. The potential of 3D-FISH and super-resolution structured illumination microscopy for studies of 3D nuclear architecture: 3D structured illumination microscopy of defined chromosomal structures visualized by $3 \mathrm{D}$ (immuno)-FISH opens new perspectives for studies of nuclear architecture. Bioessays 34, 412-426. 


\section{SUPPLEMENTARY FIGURES}

a
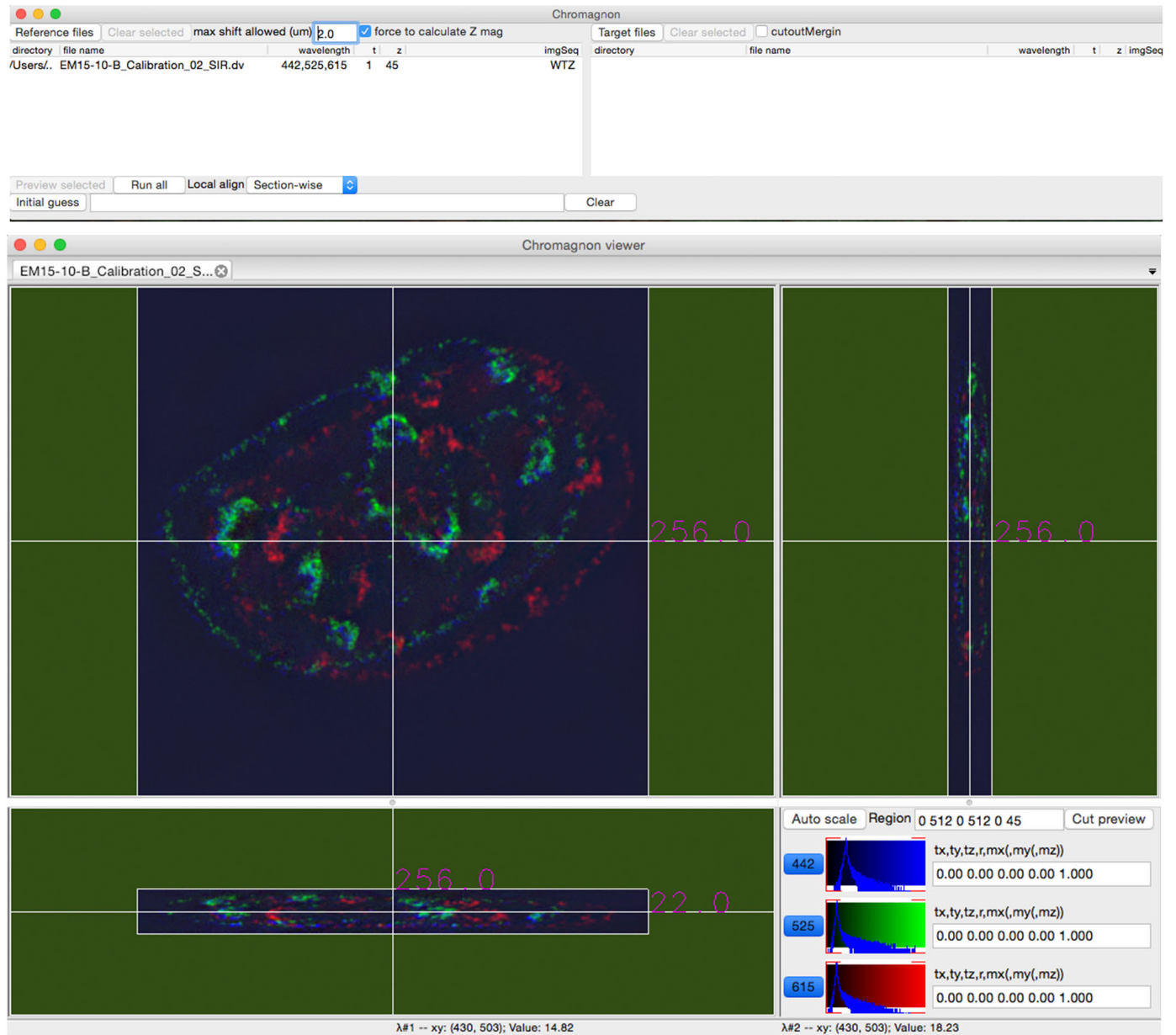

Auto scale Region $05120512045 \quad$ Cut preview
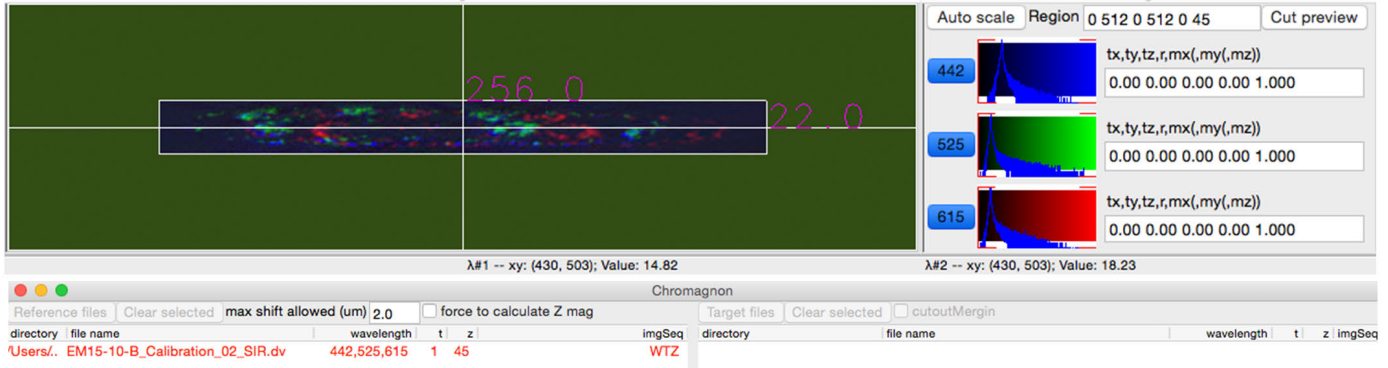

2.- xy: (430, 503): Value: 18.23

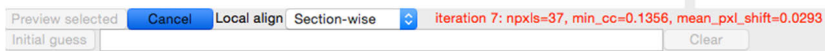

b

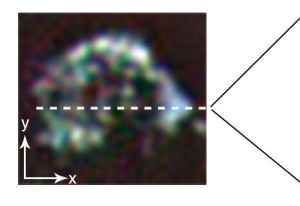

$\mathrm{X}-\mathrm{Z}$ line profile after 2D X-Y alignment

$X-Z$ line profile after 3D X-Y-Z alignemt
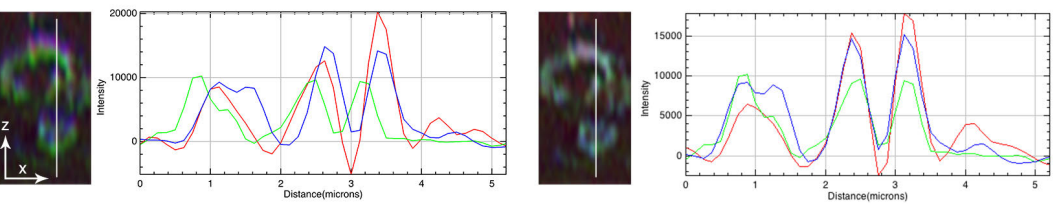

Supplementary Figure 1 | Adaptive image registration for 3D channel alignment.

(a, top) Screenshot of processing a 3D calibration data set with preview in Chromagnon.

View of software after alignment (a, bottom). The software allows the export of calculated parameters for later use, loading a previously exported parameter file, or continuing immediately to start a batch processing alignment with the currently loaded parameter. (b) Inset from a (after alignment), showing intensity line profiles in the z-dimension of an orthogonal image section after only 2D $x y$ alignment vs. 3D $x y z$ alignment. As shown by the difference in peak overlaps of the profiles, 3D alignment is required to have no residual $z$-shift in the image registration. 
a

\section{Single file workflow}

Repeat

Image Data import

7

Setup and execution of segmentation

Setup and execution of measurement

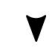

Data export

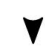

Data visualization

$$
\checkmark
$$

Biological analysis

\section{Batch-oriented workflow}

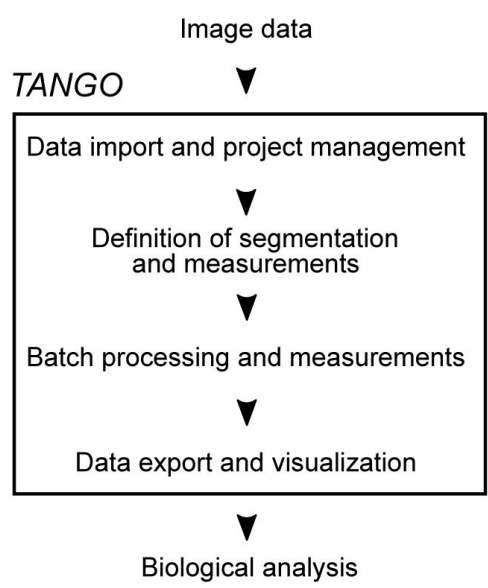

b

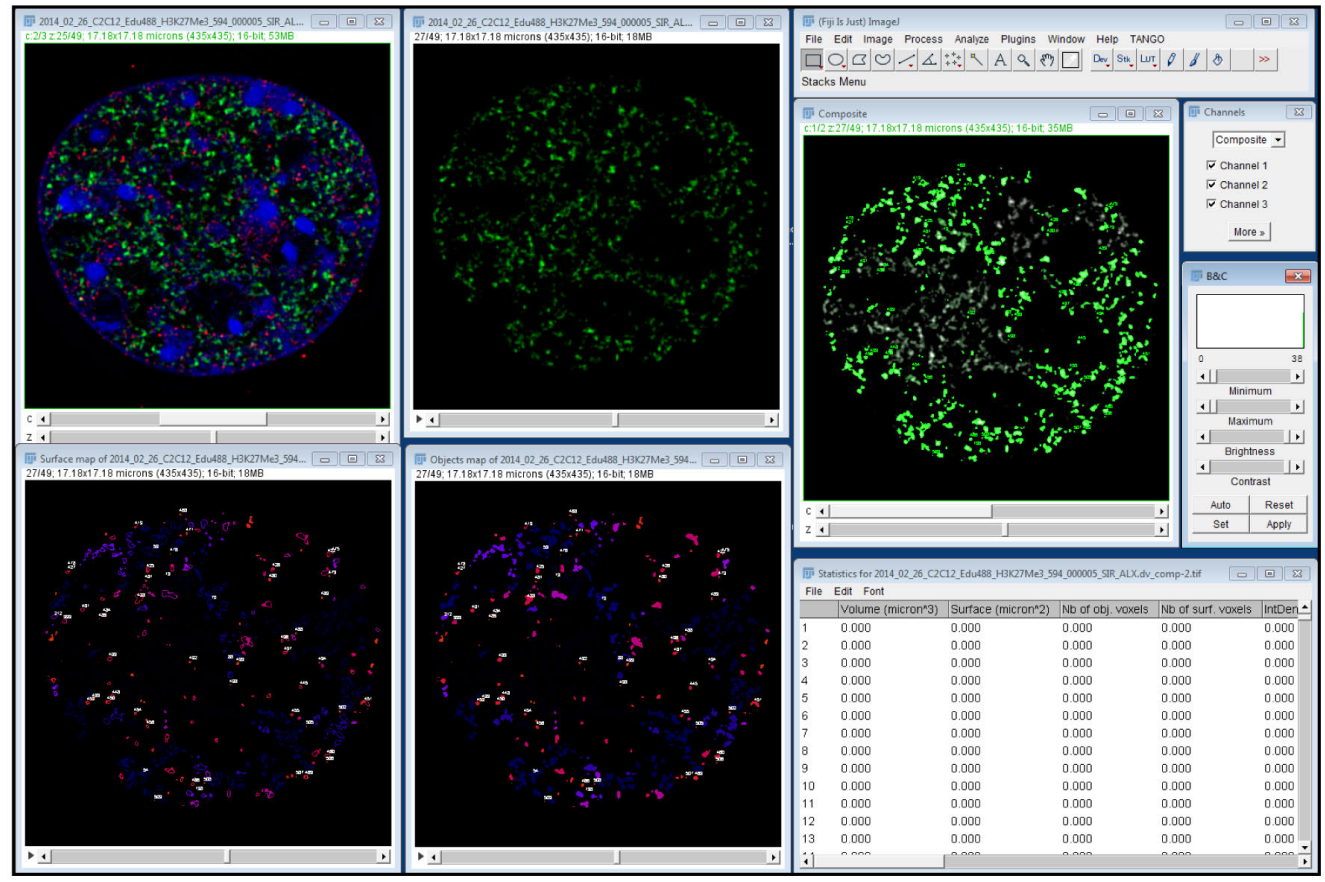

C

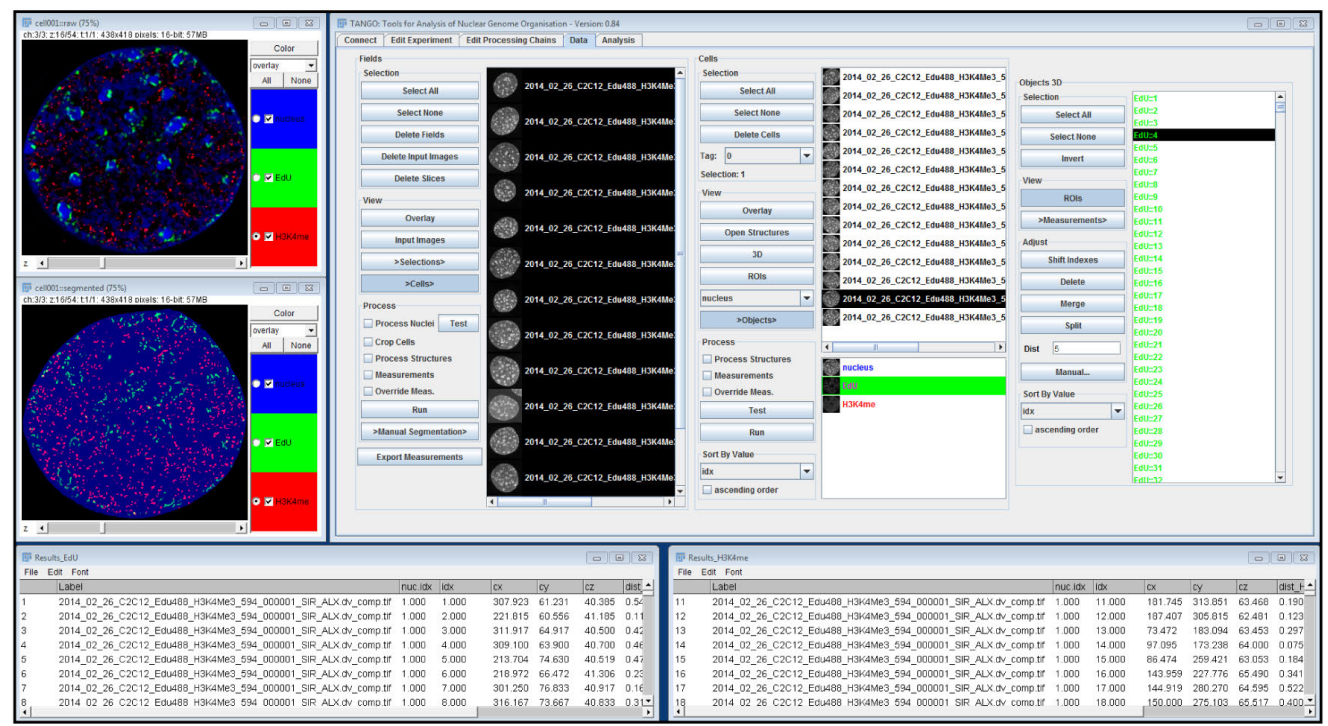


Supplementary Figure 2 | Comparison of segmentation and quantitative analysis of single vs. batch processing. (a) Workflow of single and batch-processing analysis. In single processing, individual image stacks are manually segmented and analyzed in an iterative manner. In batch mode, emphasis is placed on the definition of segmentation parameters and measurements with subsequent automated segmentation and analysis of all image data of a project. (b) Example for single processing in Fiji. Segmentation of each channel in the input data set needs to be done sequentially, in an iterative manner. (c) Example program for batch image analysis in TANGO. All data sets of a given project are analyzed in one run, following defined parameters. Pooled results of all data in one project can be exported and analyzed at once.
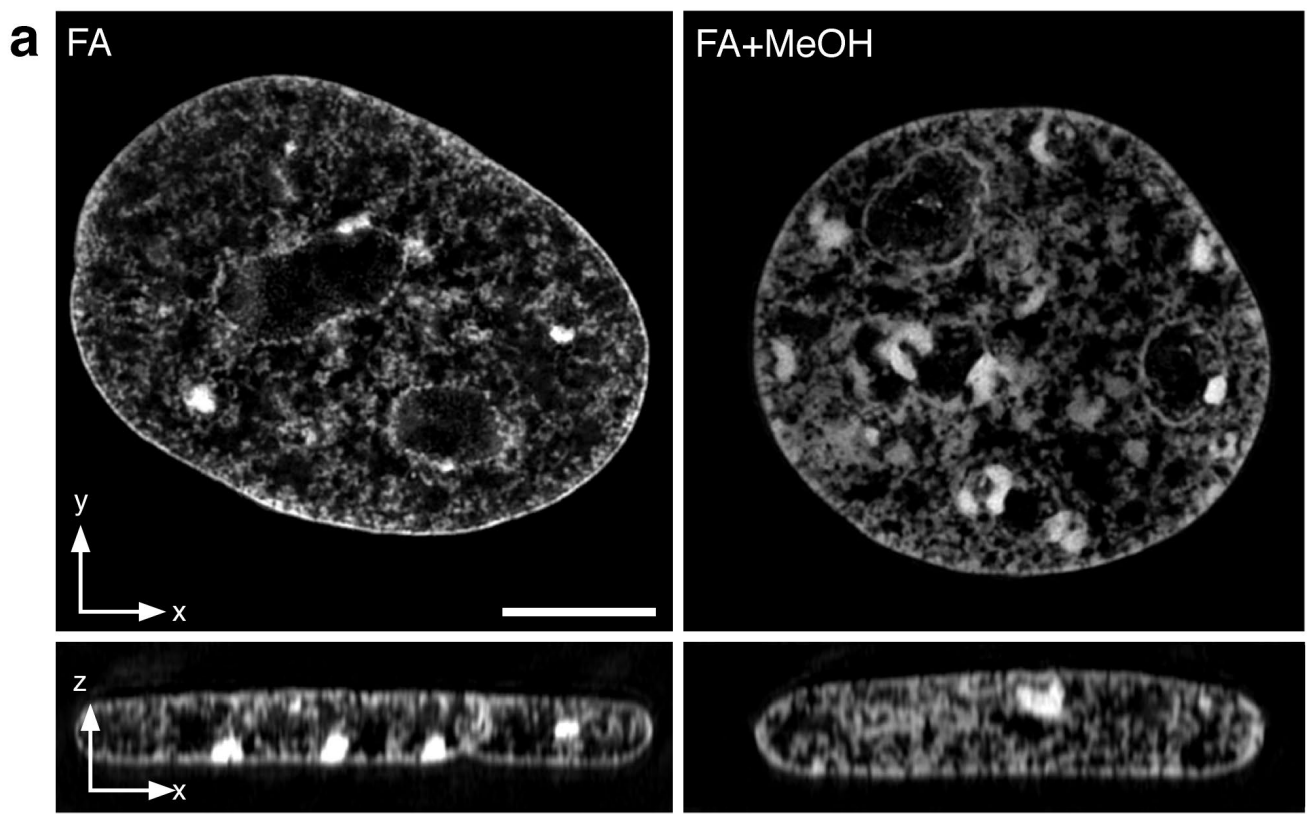

b

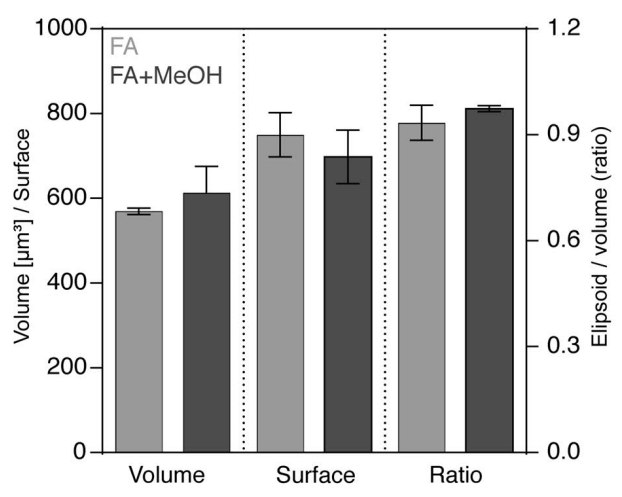

c

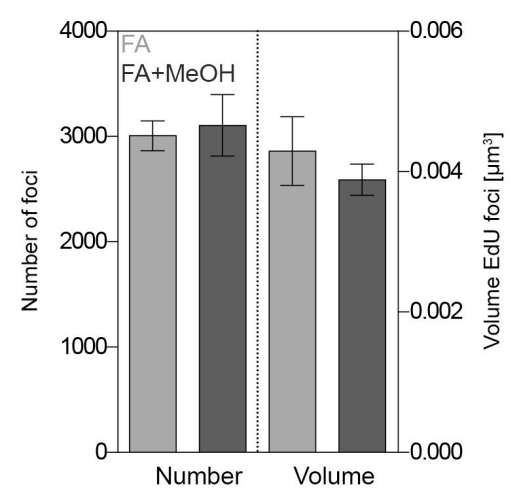

Supplementary Figure 3 | Comparison of nuclear morphological features with different fixation methods. (a) Images of DAPI stained nuclei after $2 \%$ formaldehyde (FA) or $2 \%$ formaldehyde followed by methanol $(\mathrm{FA}+\mathrm{MeOH})$ fixation. Nuclear volume, surface and morphology (ratio ellipsoid/volume) (b) as well as average replication foci number/volume (c) do not differ significantly between the two fixation methods. Scale bar, $2.5 \mu \mathrm{m}$. 